\title{
The Ultrastructure of the Nuclear Envelope of Amphibian Oocytes: A Reinvestigation
}

\author{
I. The Mature Oocyte
}

\author{
Werner W. Franke and Ulrich Scheer \\ Division of Cell Biology, Institute of Biology II, University of Freiburg i. Br., Germany \\ Received May 20, 1969, and in revised form August 22, 1969
}

\begin{abstract}
In order to review the contradictory statements on the ultrastructure of the nuclear envelope, a study was undertaken combining section and negative staining electron microscopy on manually isolated oocyte nuclei and nuclear envelopes from six amphibian species including Anura as well as Urodela.

The appearance of the negatively stained isolated nuclear envelopes is described in detail and the dependence on the preparation conditions used is emphasized. Pore complex structures such as pore perimeter, central granule, annular components, internal fibrils, and annulus-attached fibrils could be identified by both techniques, negative staining and sections.

Comparative studies show that no marked differences exist in the structural data of the nuclear envelope among the investigated amphibians and the significance of the structural components is discussed. A model of the nuclear pore complex based on the findings of the present investigation is presented.
\end{abstract}

To obtain an insight into the mechanisms involved in nucleocytoplasmic interaction, a thorough knowledge of the exchange sites of macromolecules between these two compartments is a prior condition. Since Callan and Tomlin (II) have shown that the nuclear envelope is interrupted by discontinuities of constant size and characteristic structural features, it is widely accepted that such nuclear "pore complexes" $(80)$ are the gateways through which nucleocytoplasmic interactions chiefly take place. This is at least in line with the evidence hitherto accumulated (for reviews, see e.g., $32,71)$. Considerable research on the structure and function of the nuclear membrane has been concerned with oocytes, particularly with amphibian oocytes $(20,29-34$, $36,37,43,55,64,66,74,79,82,84)$. Despite the large number of ultrastructural studies on the nuclear pore complex, there exist many astonishingly diverse models describing the spatial and functional relationship of the annulus and the membranes limiting the nuclear pores (e.g., 2, 5, 32, 34, 43, 55, 62, 68, 77, 81, 82). Furthermore, recent articles (e.g., 33, 34) on structural details of the amphibian oocyte nuclear pore 
complex have described a negative staining appearance of the pore shape, the pore complex interior, and the annulus which is totally different from that known from other cells and also from the same oocyte material after employing other methods. In order to elucidate further the structural nature of the nuclear pore complex and to review the contradictory reports on pecularities of the amphibian nuclear envelope, the present electron microscopical study was undertaken combining both negative staining and section work.

\section{MATERIALS AND METHODS}

The Urodela and Anura used in this work, Triturus alpestris Laur., Triturus helveticus Raz., Rana temporaria L., Bufo bufo L., and Bombina variegata L. were collected in ponds near Freiburg (Black Forest). Clawed toads (Xenopus laevis Daudin) were a gift from the Institute of Zoology, University of Freiburg. Ovaries were removed from freshly decapitated animals and immediately brought either to a medium made up of $0.1 \mathrm{M} \mathrm{KCl}$ and $0.1 \mathrm{M} \mathrm{NaCl}$ in a ratio of $5: 1(12,34)$ or to a medium of the same composition but with the addition of 2 or $10 \mathrm{mM} \mathrm{MgCl}$ or $\mathrm{CaCl}_{2}$. In some experiments a medium consisting of $0.2 \mathrm{M}$ sucrose, $2 \%$ gum arabic, $0.02 M$ Tris buffer, adjusted to $\mathrm{pH} \mathrm{7.2,} \mathrm{and} 4 \mathrm{~m} M n$-octanol, i.e., a medium well recommended for isolating nuclear envelopes from tissues (23), was employed for comparison. Preparations using pure Tris-buffered $(0.02 M, \mathrm{pH} 7.2)$ hypotonic sucrose solutions $(0.1$ or $0.05 M)$ were also tried.

Nuclei of fully mature eggs were isolated according to the method of Callan and Tomlin (II) and more or less quickly transferred onto a Formvar-coated grid. After most of the solution had been drawn off, the flattened nuclear envelope was fixed for 15 min at $4 \mathrm{C}$ by adding a drop of $1 \% \mathrm{OsO}_{4}$, buffered to $\mathrm{pH} 7.2$ with $0.05 \mathrm{M}$ cacodylate. Then it was washed twice in bidistilled water and negatively stained with $2 \%$ phosphotungstic acid (PTA), adjusted with $\mathrm{NaOH}$ to $\mathrm{pH}$ 7.2. In certain preparations particular care was taken that the whole procedure from tearing the oocyte up to the addition of the fixative did not last longer than 1 min. A $2 \%$ ammonium molybdate solution, adjusted to $\mathrm{pH} 7.2$, and a $1 \%$ uranyl acetate solution, adjusted to $\mathrm{pH} 4.5$, were also used as negative staining agents in some experiments.

Furthermore, isolated oocytes and nuclei were directly fixed in $4 \%$ glutaraldehyde, $0.05 \mathrm{M}$ cacodylate-buffered to $\mathrm{pH} 7.2$, for 3 hours in the cold, washed thoroughly with buffer and osmicated for 2 hours with $1 \% \mathrm{OsO}_{4}$, buffered in the same way. Dehydration was carried out by Sitte's acetone vapor method (70) or through ethanol series. The material then was embedded in Araldite and sectioned on a Reichert ultramicrotome with diamond knives. Sections were poststained with uranyl acetate and lead citrate. Micrographs were made with a Siemens Elmiskop IA. The calibration of the magnification indicator was routinely controlled using grating replicas with different spacings.

\section{RESULTS}

Negative staining

The only electron micrographs of negatively stained nuclear envelopes from mature oocytes hitherto published are those presented by Gall (32-34). In his pre- 
parations of nuclear membranes from Triturus viridescens and Rana pipiens, the pores revealed a $60 \AA$ thick margin as the only prominent negatively stained structure. Electron-transparent structures within the pore lumen such as the central granule or the internal fibrils as well as an annulus were not observed. In all oocyte nuclear envelopes from the amphibians investigated in the present study, however, annuli as well as internal pore structures could be observed as constant features, provided that a proper isolation procedure had been employed. It was noted that addition of bivalent cations as $\mathrm{Mg}^{2+}$ or $\mathrm{Ca}^{2+}$ to the pure alkaline salt medium as well as shortening of the duration of the entire isolation preserved more of the pore-associated material. Figs. 1 and 2 present the negative staining (PTA) of a nuclear envelope from a mature Alpine newt oocyte that had been isolated in the presence of $10 \mathrm{mM} \mathrm{MgCl}$ as quickly as possible, i.e., within $1 \mathrm{~min}$ from the moment when the nucleus was freed until the fixation. In such nuclear envelopes the margin of the pores is almost totally obscured by the well-preserved annulus material and other pore complex-associated material which is at least partially fibrillar (Fig. 2). At higher magnification the pore complex-associated strand-like material often seems to interconnect adjacent pores (Figs. 2, 20, and 21). A similar aspect of the amphibian oocyte nuclear pore complexes can also be visualized in preparations in which a $2 \mathrm{mM}$ concentration of bivalents was used. Fig. 4, on the other hand, shows a nuclear envelope isolated from an oocyte of the same newt again in the presence of $10 \mathrm{mM} \mathrm{MgCl}$, but in this case the isolation had taken more than $3 \mathrm{~min}$. While some of the pore complex-associated material has obviously been detached, the annulus can still be detected as well as inner pore structures, such as the central granule and the inner pore fibrils. The pore complex structure, however, was greatly altered when negatively stained after isolation in a pure alkaline medium without any bivalent cations and, especially, when the isolation procedure had taken a relatively long time. Fig. 5 illustrates such a nuclear envelope after a 10 -min isolation in a medium containing solely alkaline ions. The appearance of such "empty" pore complexes resembles closely that shown by Gall (34) in that only the sharply delineated outline of the pore lumen is negatively contrasted from the thinly PTA-covered envelope and the heavily PTA-filled pore lumen. Neither an annulus nor any remarkable internal pore structures can be recognized. Thus, something like a progressive differential dismantling of the pore complex seems to take place during the course of such a preparation. While studies on the pore-attached material would require stabilization by added bivalent cations (see also 54, 67), it is, on the other hand, apparently advantageous to prefer a prolonged influence of a pure

FIG. 1. Nuclear envelope isolated from a mature Triturus alpestris oocyte in the presence of $10 \mathrm{mM}$ $\mathrm{MgCl}_{2}$ (less than 1 min until fixation), fixed with $\mathrm{OsO}_{4}$ and negatively stained with phosphotungstic acid $\times 43,000$. 


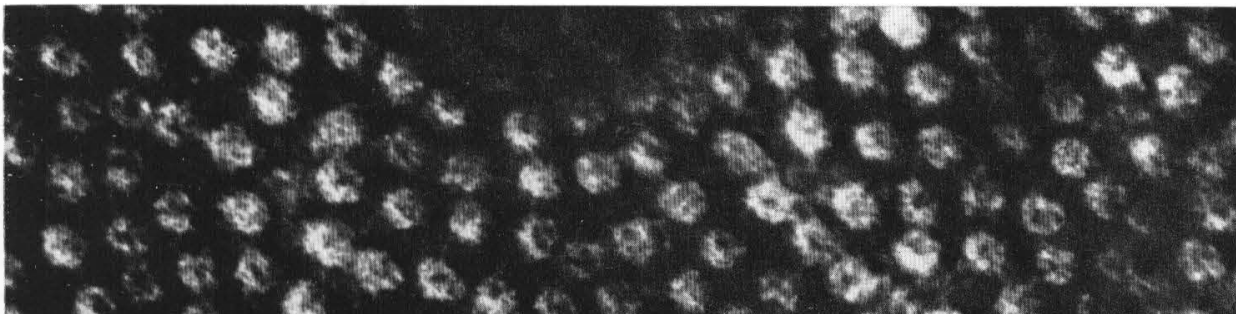

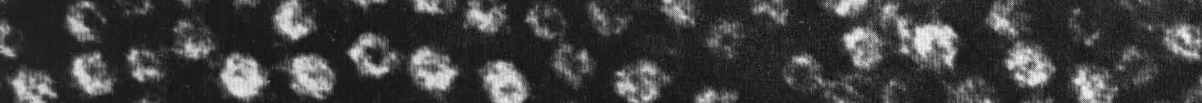
4. 5 .

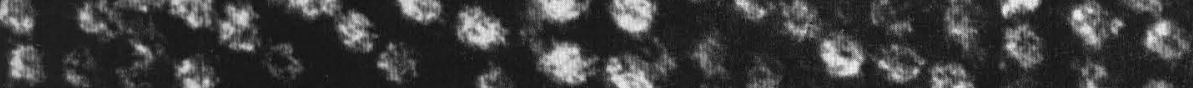

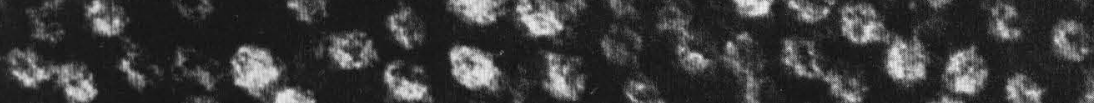

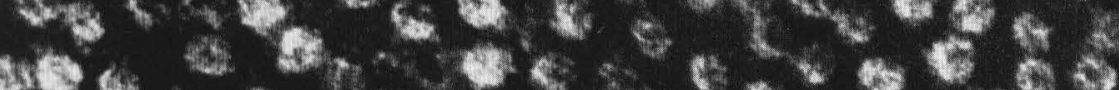

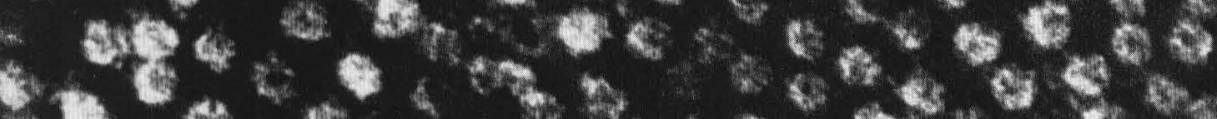

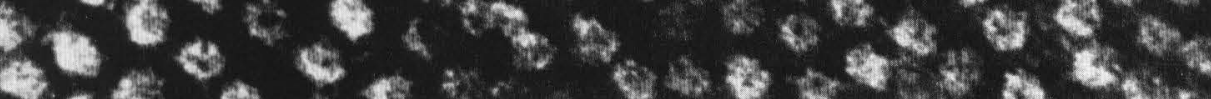

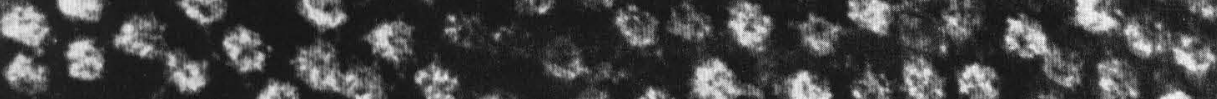

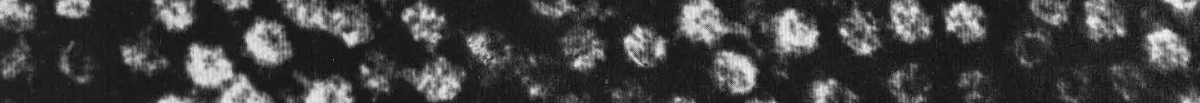

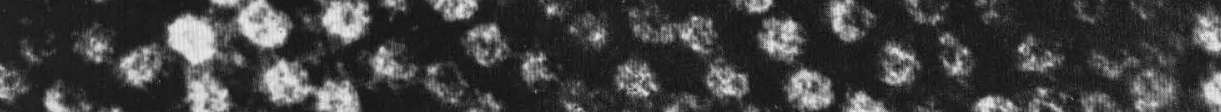

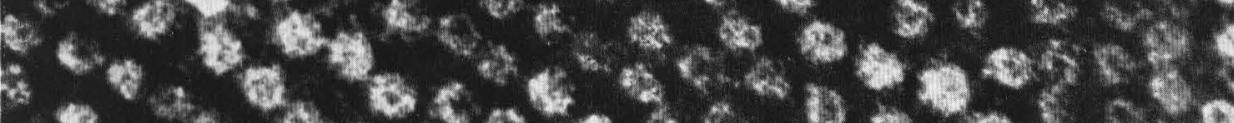

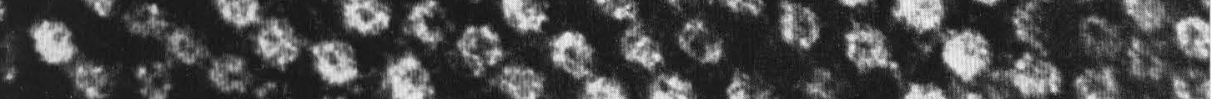

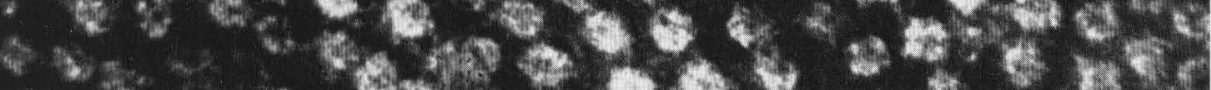

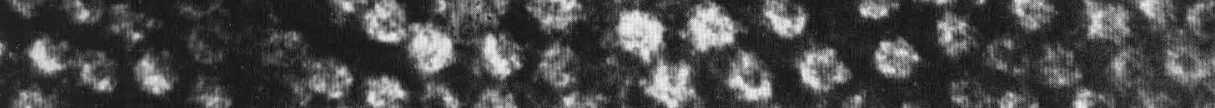
B.

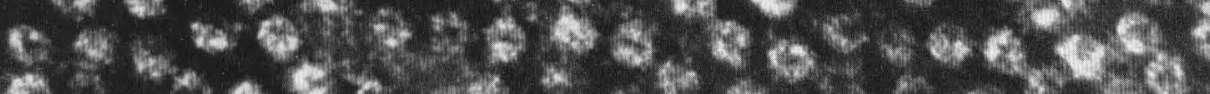

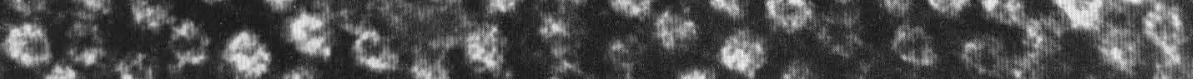

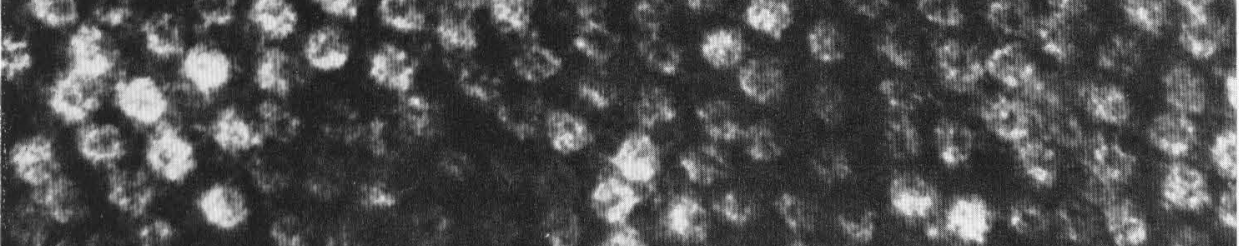

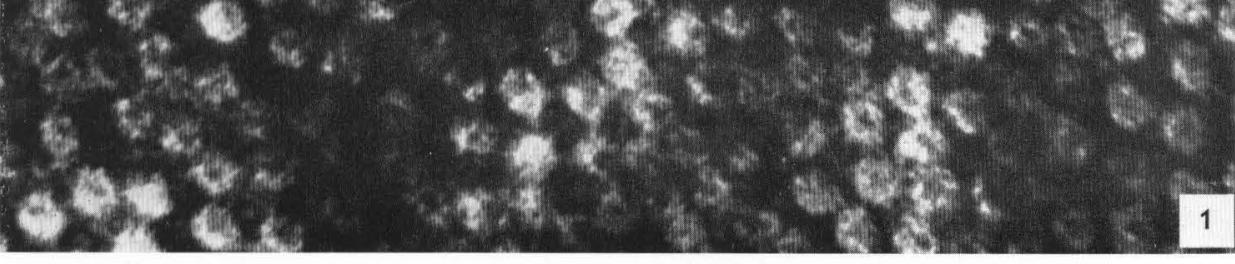


alkaline medium when working, e.g., on the pore dimensions. In this connection it might be noteworthy that sometimes slightly different states of pore-associated material could be seen in the same nuclear envelope, thus indicating that local differences, be they biologically or artificially caused, can exist in different parts of an envelope. Short wash treatment of the envelopes with bidistilled water prior to fixation frequently causes a tearing into smaller fragments (Fig. 3). Negatively stained envelopes isolated in the medium that is routinely used in our laboratory for mass isolations of nuclear membranes from plant and animal tissue $(23-25,88)$ displayed the pore-associated material so abundant that the envelopes appeared to be sprinkled by a biological contamination of material adhering to the envelope. Under such conditions the pore complexes barely could be identified. This increased preservation of annular and other pore material by a sucrose-containing medium recalls the early observation made by Bovey (cited by Wilkins, 83) on the stabilization of the annuli of nuclei from Gregarina acridiorum. The pore complexes, however, appeared not to be sufficiently preserved, in that they did not show the annulus and other poreassociated material when isolated in a hypotonic sucrose solution (Fig. 9),

The described dependence of the nuclear pore complex appearance on the isolation conditions also was found to hold for the oocytes of the other investigated amphibian species. The extent of such artificially induced alterations of the pore complex structures is illustrated in a series of micrographs showing nuclear membranes from mature frog eggs. For instance, much of the annulus material is preserved in the preparation shown in Fig. 10 whereas such material is totally absent in the envelope of Fig. 11 which has been prepared from the same ovary but was exposed longer to the medium. Furthermore, in some of the nuclear envelopes intermediate stages of loss of the pore-associated material could be found (e.g., Figs. 7, 21, 30, and 36-39); here the distinctly visible pore margin is accentuated by separate "white" dots interpreted to represent core remnants of the globular subunits of the annulus while the more amorphous and the fibrillar material seem to have disappeared.

The situation is quite similar in toad eggs (Bufo bufo; Fig. 12) and eggs from Bombina variegata (Fig. 13) as well as in Xenopus laevis oocytes (Fig. 14) and those from the Swiss newt (Triturus helveticus, Fig. 15). Thus with respect to the structural lability of pore complex material under the conditions described, no specific dif-

FIG. 2. Similar preparation as in Fig. 1. Annulus material is well preserved and appears to be associated with finely fibrillar material. $\times 66,000$.

FIG. 3. Torn nuclear envelope piece of a Triturus alpestris oocyte after isolation in pure alkaline medium and washing with bidistilled water prior to fixation. Note the fragmentation borders running around the pore complexes, thus indicating that the pore complexes are loci of remarkable rigidity (lower arrow). Thin fibrils of unidentified nature are seen as connecting the envelope fragments (upper arrows). $d A$, detached annulus lying separately on the supporting film. $\times 59,000$. 

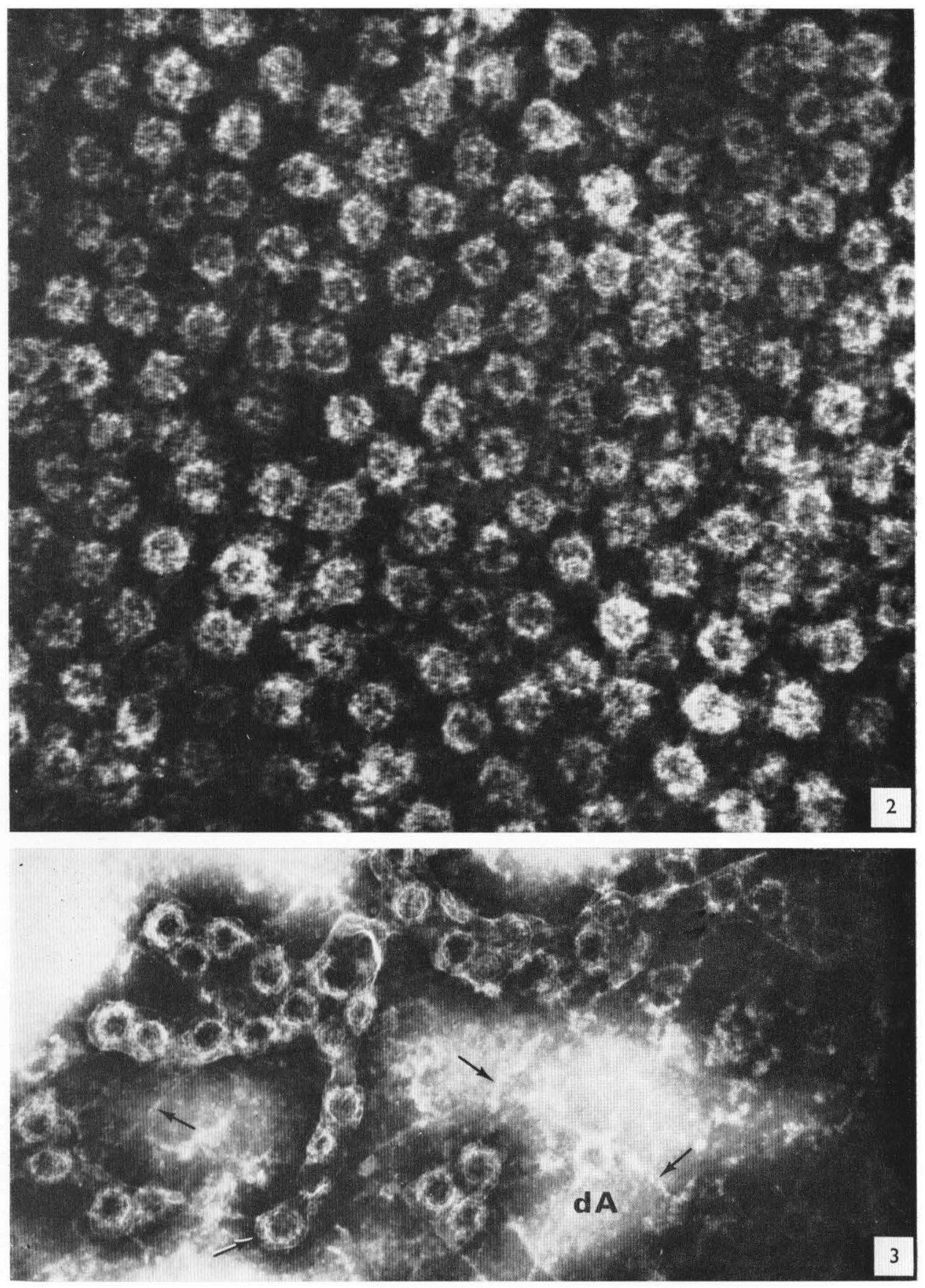
ferences were observed between the mature oocytes of the different amphibians under study.

Progressive loss of pore complex-associated material, however, is not the only artifact which can occur during the course of the Callan-Tomlin preparation. Frequently, when no stabilizing bivalents were present and the procedure lasted relatively long, myelinization structures could be encountered on the nuclear envelope revealing either the classic lamellar appearance (e.g., Fig. 8) or more cylindrical configurations ("myelinization craters", Fig. 5), The apparent mechanical stress exerted on the envelope during the manipulations, and most likely during the spreading on the grid, can furthermore result in a fragmentation of the envelope sheet (Fig. 3). This effect might, for instance, be seen from Fig. 3 where small envelope fragments can be found associated with unidentified, strand-like material. In this connection, one should note that fragmentation of the envelope sheet, which takes place more rapidly when washing the envelopes with water, partially circumscribes the pore complexes so as to produce nearly isolated pore complexes, a phenomenon that has been mentioned for nuclear membranes isolated from plant and other animal material as well $(25,46,57$, 92). PTA-repellent doughnuts with an inner diameter in the $600 \AA$ range also can be occasionally found (Fig. 3) and are interpreted as "detached annuli" according to Gall (34).

The considerable stretching of the nuclear envelope sheet obviously can cause a deformation of the circular pore to a more elongated, ellipsoidal shape (Figs. 6, 9, and 31-35). In the particular envelope piece shown in Fig. 6 the deformation axes, i.e., the longer axes of the elliptic pores, are parallel to each other and indicate the direction of the stretching process. Such distortions quite often bring about a diversity of various pore shapes such as polygonal, wrinkled, or even pyriform ones (Figs. 6-9, 13, and 31-35).

Since some recent papers have stressed the special validity of ammonium molybdate as a negative staining agent that can be used in concentrations isotonic to cisternal membrane systems, such as microsomes $(59,60)$, we made some preparations using a neutralized $2 \%$ solution of ammonium molybdate with and without a preceding fixation. The appearance of the pore complexes, however, could not be significantly

Fig. 4. Appearance of a nuclear envelope from the same ovary as that shown in Figs. 1 and 2, isolated in the presence of the same $\mathrm{Mg}^{2+}$ concentration but after prolonged exposure to the medium during the isolation procedure. $\times 51,000$.

FIG. 5. Nuclear envelope from the same ovary as shown in Fig. 4 but isolated in a pure alkaline medium $(10 \mathrm{~min})$. No annular or internal stuctures can be discerned in the pore complexes. Note the myelinization craters (arrows). × 56,000.

FIG. 6. Envelope isolated under conditions similar to those of Fig. 5. The pores are parallel distorted to a more ellipsoidal shape, presumably by a directed stretching process during the spreading and drying. Note also the high degree of myelinization in such a preparation. $\times 45,000$. 

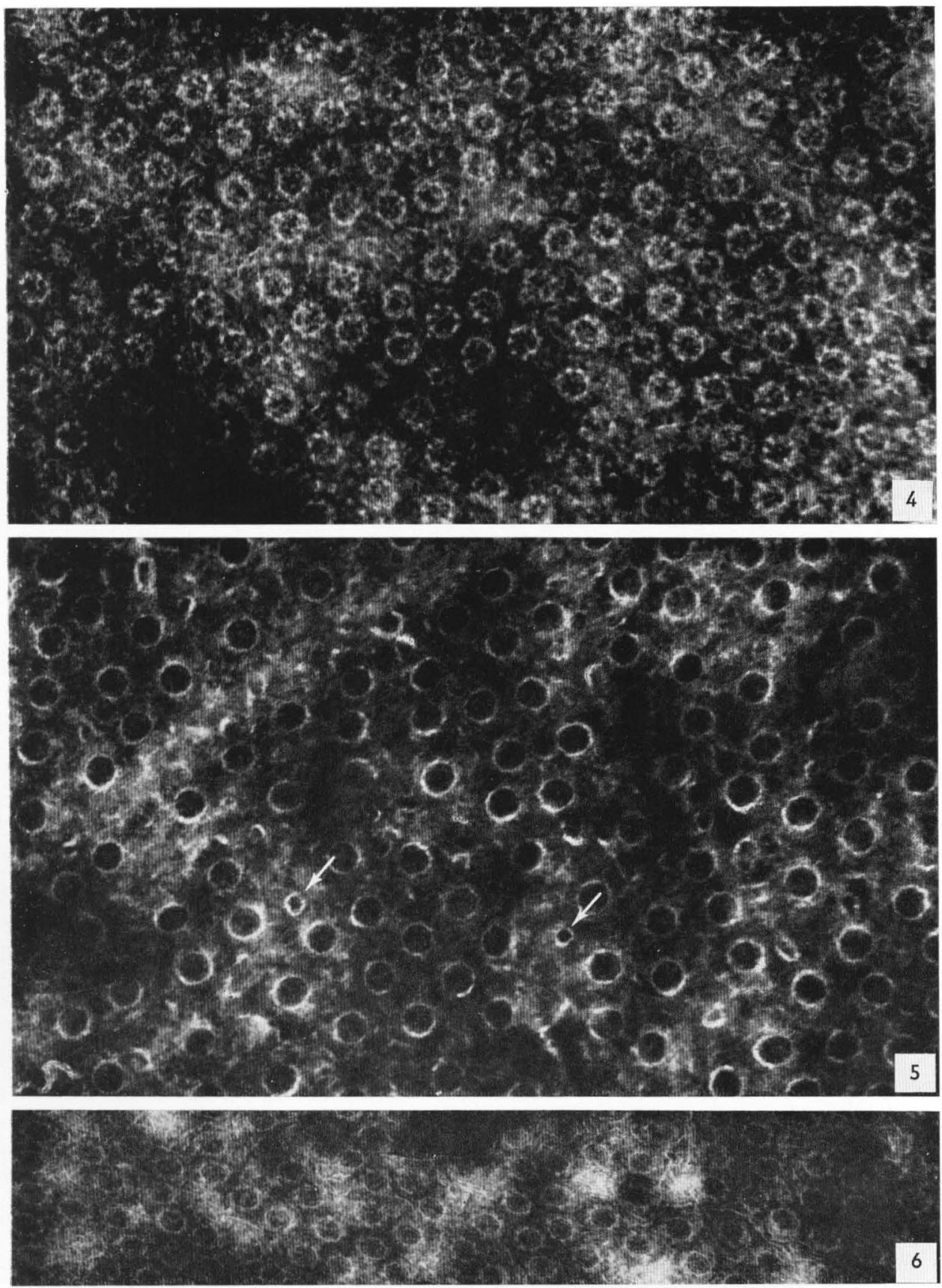
distinguished from that obtained by using PTA (Fig. 16). Staining with the slightly acid $1 \%$ uranyl acetate was found to be insufficient in that it produces extensive membrane disintegration.

Summarizing the ultrastructural details of the negatively stained pore complex of amphibian oocytes under the different preparation conditions, one can discern the following nonmembraneous substructural constituents.

1. Granular subunits of the annulus. These are globules with diameters of 100 to $180 \AA$ most clearly seen in preparations in which a certain portion of the poreassociated material seems to be lost (Figs. 4, 7, 16-18, 21, 30, and 36-39). Markham's rotational analysis (49) shows them to be arranged with regular spacings in a radial symmetry which is predominantly 8 -fold (Figs. 17 and 18).

2. About 30 A broad "annulus-attached fibrils." These can often be observed suggesting an origin from the pore margin or from the particulate annular subunits (Figs. 2, 4, 10, 13, 20, 21, 25, and 36). The fibrils are sometimes studded with small granules about $50-90 \AA$ in diameter (e.g., Fig. 25). In a great many preparations they can be discerned as extending to the annuli of neighboring pore complexes (e.g., Figs. 2, 10, 20, 21, and 36), but this may be caused artificially by a random settling of the fibrils onto the envelope sheet during the preparation.

3. Tip-like projections. More particulate tips of material conically projecting from the annulus into the pore lumen (e.g., Figs. 17 and 28) are known to be a widespread feature of pore complexes of isolated nuclear envelopes ("peripheral granules" sensu $92,23-25,88)$ as well as of isolated annulate lamellae (68). They seem to originate from the annular subunits and thus to be distributed along the pore circumference in a symmetry corresponding to the 8-fold symmetry of the annular globules (Fig. 17).

4. Internal fibrils within the pore lumen. These fibrils are equal in size and negative staining appearance to the annular fibrils (Figs. 21-24, 29, and 36). Their image often suggests an origin from the annulus or the pore margin. Frequently the internal fibrils take part in the formation of more compound inner pore complex structures such as the "inner ring" (e.g., Figs. 23 and 24; see also 88, 92) and the radiating spokes pattern ("thread-like structures" sensu 92; e.g., Fig. 29) in which these fibrils are connected to the central granule. In some pores the internal fibrils, particularly when arranged in the "inner ring" configuration, were found to be also associated with 50-70 $\AA$ large granular particles as is the case in the annular fibrils.

FIG. 7. Nuclear envelope of an almost mature Triturus alpestris egg isolated quickly (about 3 min) a pure alkaline medium. Annulus material is only partially preserved, but the more persistent annular subunits are now particularly well recognizable. $\times 48,000$.

FIG. 8. Typical lamellar myelinization pattern such as is frequently observable in nuclear envelopes isolated in media consisting solely of alkaline ions. $\times 45,000$.

FIG. 9. Appearance of nuclear pore complexes when isolated in a pure $0.1 M$ sucrose solution. $\times 43,000$. 

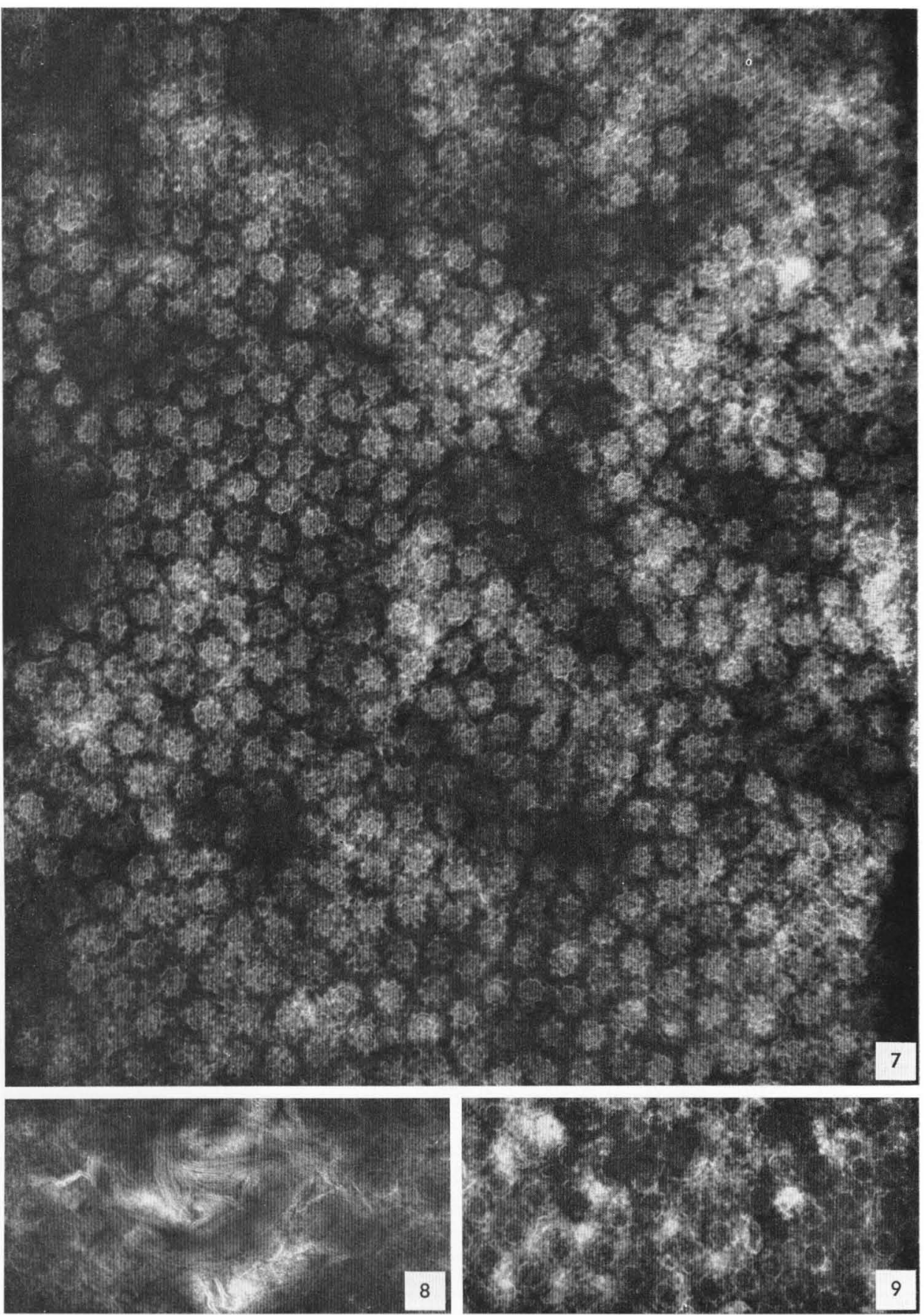
5. The central granule. The diameter of this centrally located pore complex particle was found in the negatively stained nuclear envelopes from amphibian oocytes to vary from $50 \AA$ (e.g., Fig. 22) up to about $300 \AA$ (e.g., Fig. 27). That the larger central granules were composed of smaller granular particles, as has been reported from negative staining preparations of isolated pea seedling nuclear envelopes (92) and from ultrathin sections through frog oocytes (43), was only occasionally indicated in our preparations (e.g., Figs. 21-24). A frequent feature of the central granule is its connection to the annulus or to the pore margin by thin filaments (the internal fibrils), an arrangement which often gives rise to the radiate pattern mentioned above (e.g., Figs. 27 and 29).

6. Amorphous material of the annulus. That the annulus consists not only of the particulate globular subunits but also of more diffuse material has been described by Franke (23). In the amphibian oocyte nuclear envelopes of the present study, this is seemingly the material which detaches most easily, so that it can be seen only in preparations in which precautions for stabilization had been taken.

7. The amorphous material within the inner part of the pore. In the majority of preparations moderately stained with PTA, some loose material is visualized within the pore of a diameter which is below $25 \AA$, i.e., in the order of magnitude of the size of staining grain, and which is therefore regarded as being "nonparticulate" in the sense of the resolution limit of the method used.

\section{Sections}

Numerous references have described the appearance of the sectioned nuclear envelope of amphibian oocytes after different fixation methods. Therefore in the present study we should better focus to a clarification of some of the still rather vague structural details of the pore complex as revealed by this technique. While the annulus with its subunits can be seen easily in sections tangent to the nuclear envelope, it is either ill-defined or not at all discerned in sections cut perpendicularly to the nuclear envelope (e.g., 13). Thus, micrographs of perpendicular sections of the nuclear pore complex which show clearly recognizable annular structures rarely are found in the literature. In our material it is evident from both perpendicularly and tangentially cut thin sections that the globular subunits are well preserved. The annular globules

FIG. 10. Nuclear envelope from a mature oocyte of Rana temporaria isolated in an alkaline medium without bivalent cations. The annulus-associated fibrils are seen and in some pores also the inner pore material. $\times 55,000$.

FIG. 11. Nuclear envelope from the same ovary in the same stage of maturation as in Fig. 10, but after a slightly longer duration of the Callan-Tomlin procedure. Comparison of this micrograph with Fig. 10 indicates how delicate the appearance of the nuclear pore complexes is with respect to the preparation conditions. Note the relatively intense myelinization damage. $\times 53,000$. 

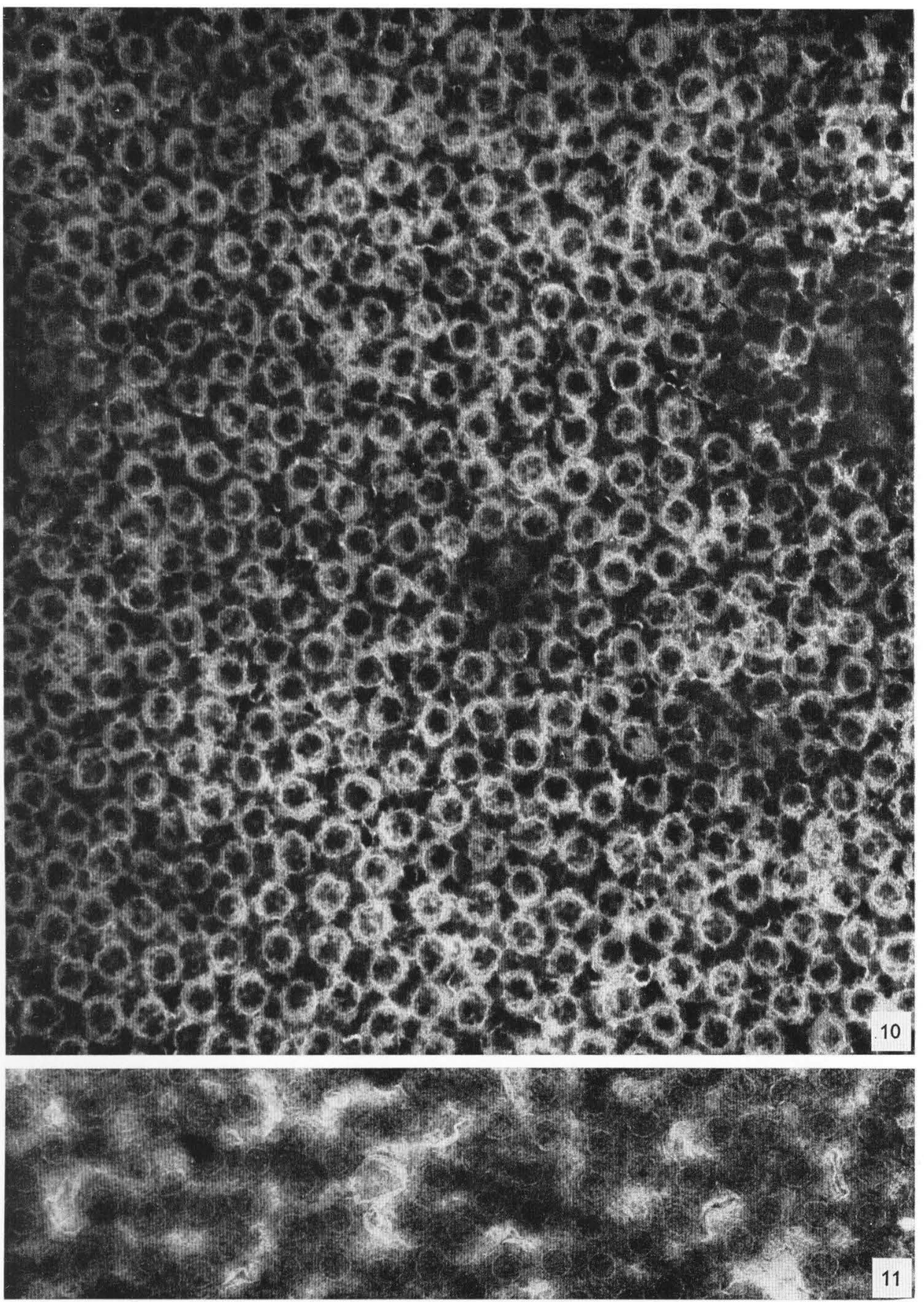
appear as electron dense, relatively distinct particles about $150 \AA$ in diameter lying upon the pore margin on either the inner and the outer side of the nuclear envelope (e.g., Figs. 19, 42-44, 46). From this simple observation some of the pore complex models proposed can be ruled out with high probability $(69,77,84)$. The number of subunits per annulus, counted from sections tangential to the nuclear surface, were predominantly found to be eight, and Markham analysis again proved a symmetrical radial pattern (Fig. 19).

A common structural feature of the pore complex in section also is the central granule and the internal fibrillar material, which sometimes seems to connect the granule with the pore margin in a spoke-like pattern (upper inset, Fig. 48).

As early as 1958 Wischnitzer (84) mentioned in previtellogenic stages of Triturus viridescens eggs the occurrence of annular "tubes" which he interpreted as composed of microcylinders running continuously through the whole length of the pore lumen.

With the fixation and embedding procedures employed today, a clearer view of these structures is attained. Fibrils up to $1 \mu$ long and about $30 \AA$ thick terminate (or originate) at the inner (nucleoplasmic) annulus of the pore complex (e.g., Figs. 41, $46,47,49$, and 50). These fibrils sometimes appear to be studded in certain spacings with small granular particles about $70 \AA$ in diameter (Figs. 40, 46, 47, 49, 51, and 52). They are arranged in a cylinder and seem to anastomose and to branch at many sites (e.g., Figs. 41, 47-49, 51, and 52). Such an interconnection of annulus-terminating fibrils composes the cylinder, which can extend into the nucleoplasm for a certain distance (Fig. 51). One often gains the impression that these fibrils end just at the globular subunits of the annulus (e.g., Fig. 46). At any rate, it seems reasonable to interpret them as being identical to the "annular fibrils" known from negative staining of the same material. A closer inspection of the fibrils sometimes reveals a somewhat helical appearance (inset Fig. 48; and Fig. 52). With a few exceptions no corresponding fibrillar structures were observed on the cytoplasmic side of the envelope.

The amorphous material of the annulus as well as that within the inner part of the pore complex can also be demonstrated, the former being more conspicuous from tangential sections, while the latter gives rise to the marked contrast within the crosssectioned pore (Figs. 42-46), the so-called "diaphragm material" described in the literature (for review, see e.g., 32). The early interpretation of Watson (81; compare also $6,32,42$ ) that the electron opacity within the pore lumen should be a consequence of section thickness and should be caused by a part of pore wall included in the section and viewed edge-on apparently cannot be further upheld (e.g., 78). Thus, when the evidence from negative staining and thin sections is considered, there is no further doubt that the interior of the pore complex is filled with some material, at least in certain physiological states of cell metabolism.

Another observation in the thin sections is the frequent occurrence of an electron 

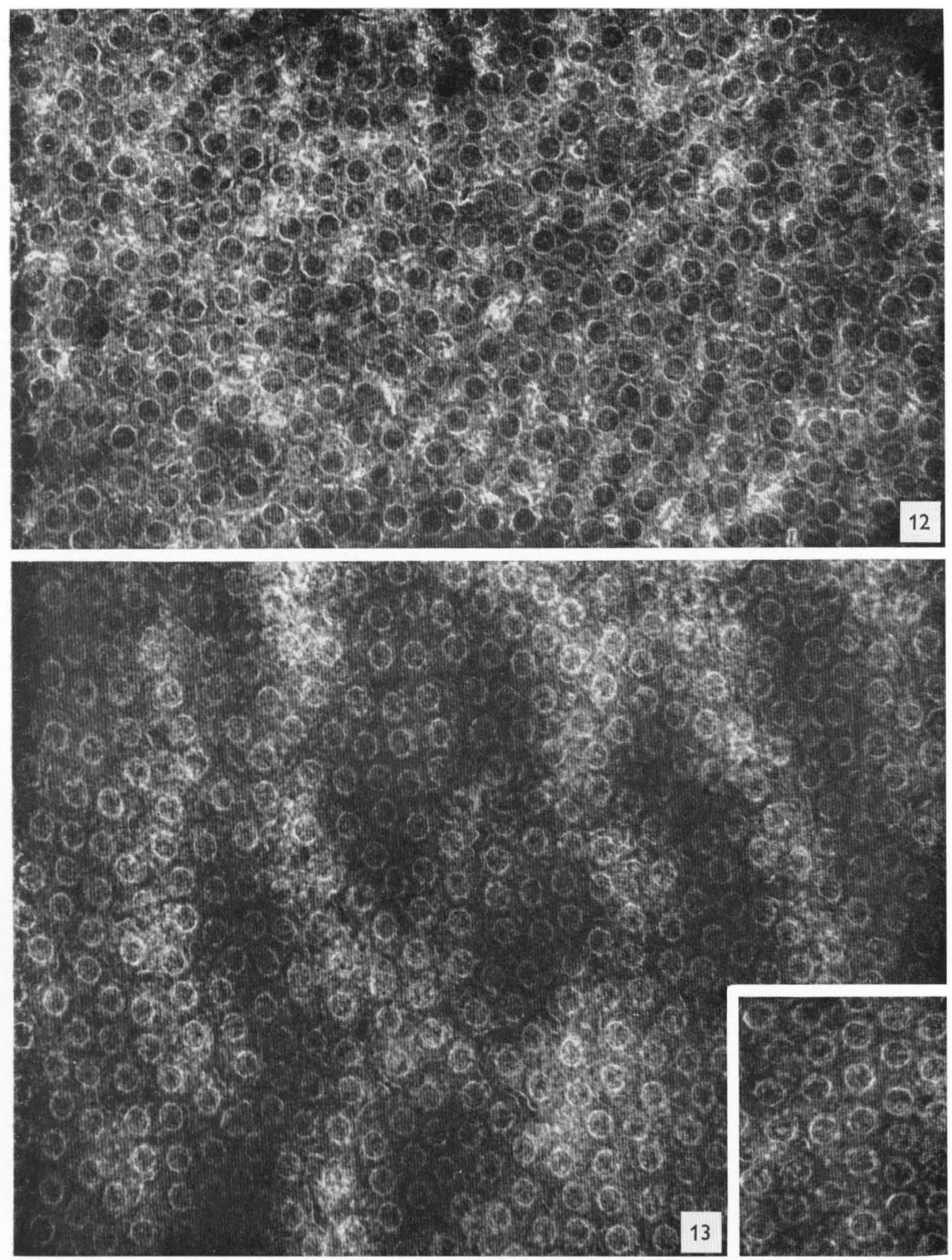

FIG. 12. Oocyte nuclear envelope of the toad Bufo bufo isolated in a pure alkaline medium, fixed, and then negatively stained with phosphotungstic acid. $\times 45,000$.

FIG. 13. Nuclear envelope from a mature oocyte of Bombina variegata prepared as described above. Inset shows some pores revealing the pore perimeter particularly clearly. $\times 45,000$; inset, $\times 50,000$. 

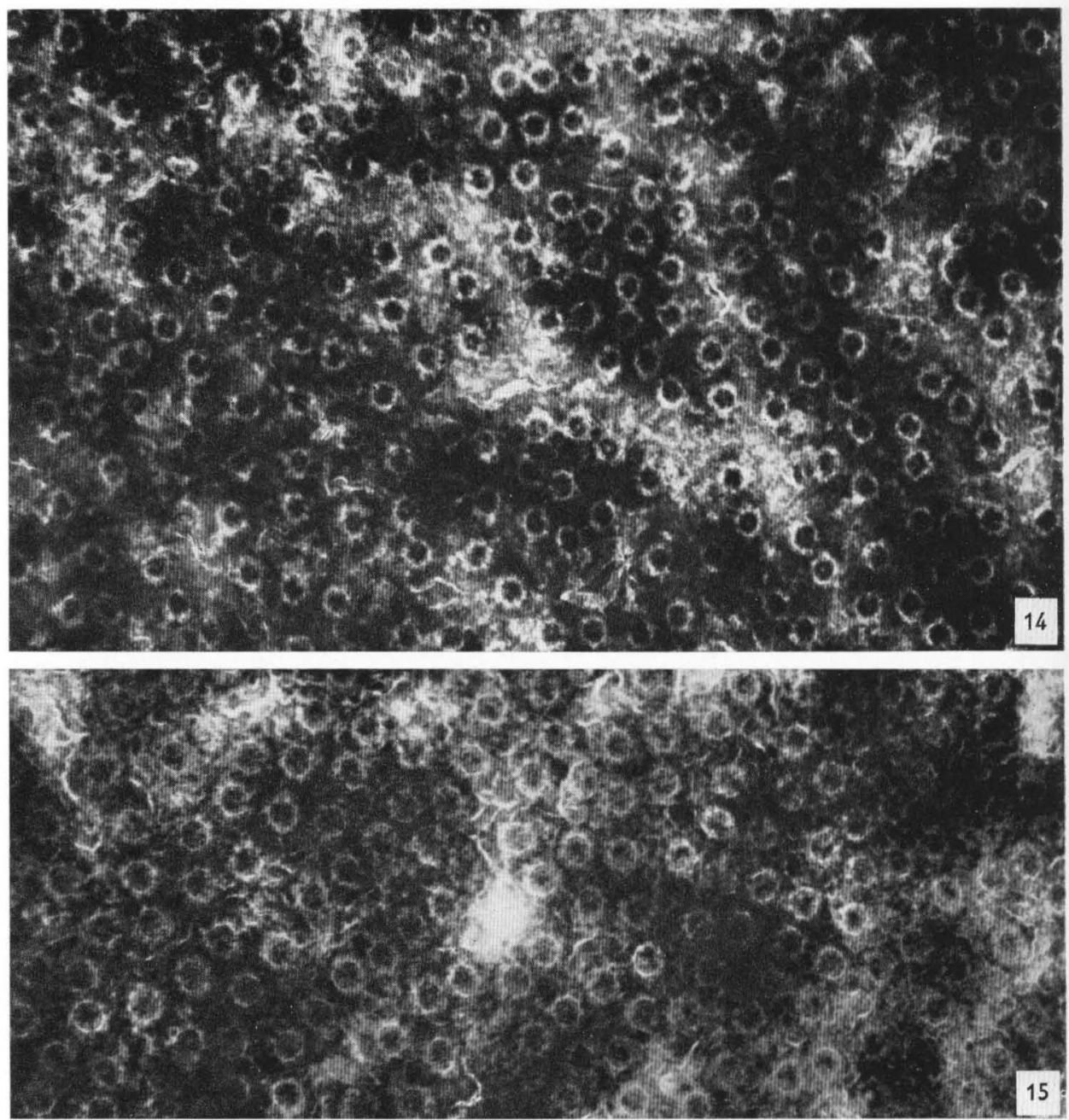

Fig. 14. Nuclear envelope of a mature egg from the clawed toad Xenopus laevis. Methods as given for Fig. 12. × 42,000.

FIG. 15. Similar preparation from Triturus helveticus. $\times 42,000$.

dense layer about $50 \AA$ thick which underlies the inner nuclear membrane (e.g., Fig. 43; compare also 68).

Frequently one can detect dense material within the cisternal space of the nuclear envelope giving either the appearance of a lamellar sheet (Fig. 45) or of separate globules (Fig. 46). Such intracisternal material so far has been observed only once by Jacob and Jurand from salivary gland nuclei of Bradysia mycorum (35). 


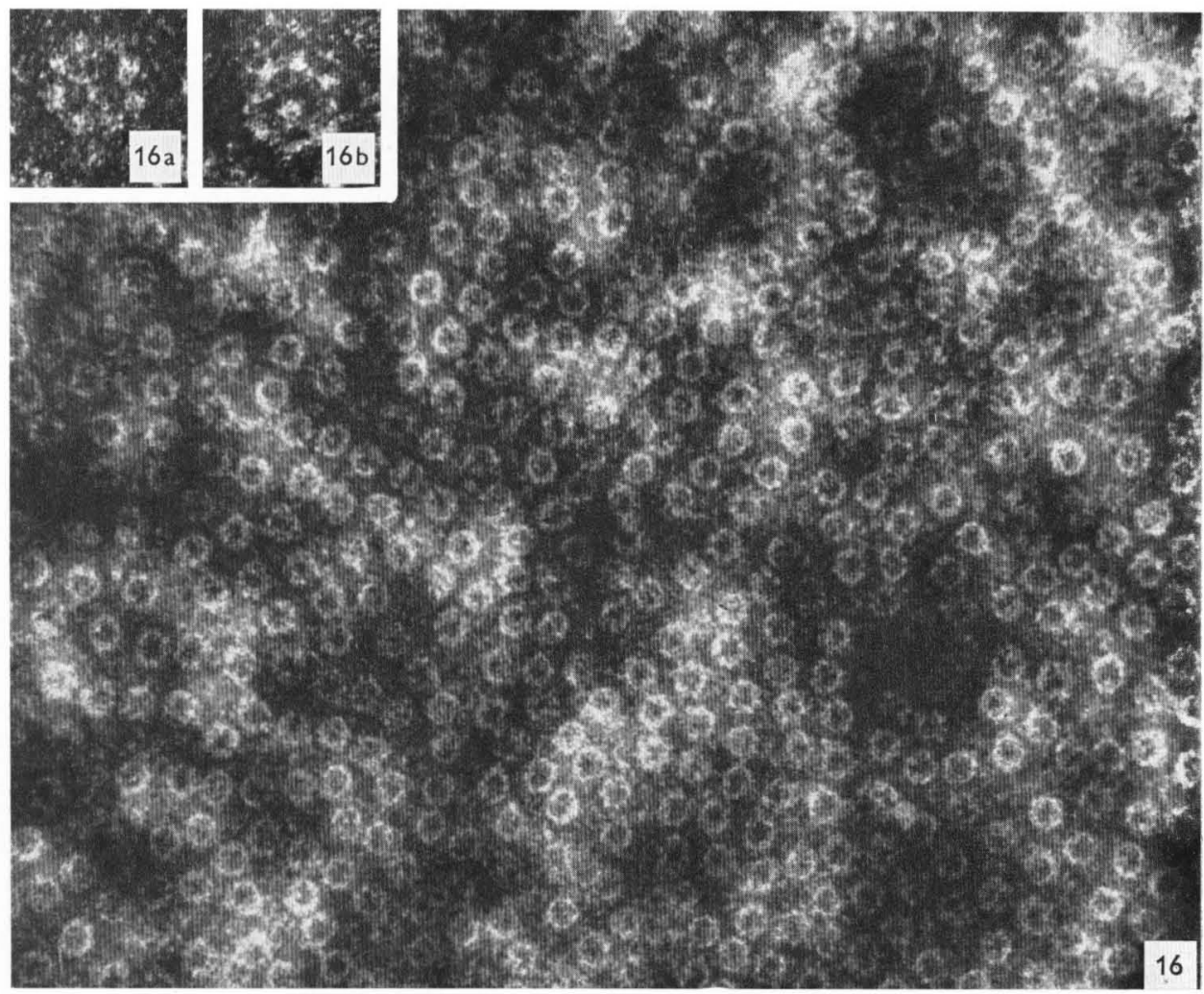

FIG. 16. Nuclear envelope from a mature Triturus alpestris egg (pure alkaline medium, short exposure), directly stained with $2 \%$ ammonium molybdate (pH 7.2). Insets show some pore complex details. $\times 39,000$; insets, $\times 110,000$.

\section{Interspecific comparison of quantitative data}

It has been mentioned above that with respect to the ultrastructural details of the pore complex and the structural stability of the pore-associated material no differences could be observed so far in mature eggs from different amphibian species. As can be seen from Table I which gives a comparison of some quantitative nuclear membrane data, there are no interspecific differences of the pore diameter, the pore frequency and the relative nuclear surface area occupied by pores. The finding of the striking constancy of the pore diameter agrees fairly with the pore diameter measurements in negatively stained eggs from Triturus viridescens and Rana pipiens (34). One should bear in mind in this context that Gall's values as well as ours refer to the "true" pore diameter, i.e., the diameter of the membrane-limited pore lumen, and thus are not to be compared with the values obtained_by using other methods (e.g., 20-691822 J. Ultrastructuré Research 
TABLE I

Interspecific Comparison of Some Quantitative Data of Nuclear Envelopes ISOLATED FROM AMPHIBIAN OOCYTES

\begin{tabular}{lllc}
\hline \multicolumn{1}{c}{ Species } & $\begin{array}{c}\text { Diameter } \\
\text { of Pore } \\
\text { Lumen }(\AA)^{a}\end{array}$ & $\begin{array}{c}\text { Pore Frequency } \\
(\text { No. of Pores } \\
\left.\text { per } \mu^{2}\right)^{b}\end{array}$ & $\begin{array}{c}\text { Percentage of } \\
\text { Nuclear Surface } \\
\text { Occupied by Pores }\end{array}$ \\
\hline $\begin{array}{l}\text { Triturus alpestris } \\
\text { Triturus helveticus }\end{array}$ & $737 \pm 67(254)$ & $50 \pm 6.4(283)$ & 21.3 \\
Bombina variegata & $742 \pm 24(130)$ & $49 \pm 8.2(17)$ & 21.2 \\
Xenopus laevis & $713 \pm 23(478)$ & $52 \pm 5.5(145)$ & 20.8 \\
Bufo bufo & $753 \pm 25(301)$ & $48 \pm 5.3(24)$ & 21.4 \\
Rana temporaria & $739 \pm 30(289)$ & $55 \pm 4.4(63)$ & 22.6 \\
\hline
\end{tabular}

${ }^{a}$ In parentheses: number of pores evaluated.

${ }^{b}$ In parentheses: square microns of evaluated nuclear surface.

55,56 ) because these studies give mostly the inner and outer annulus diameter (see detailed discussion of this problem in 32). The pore frequency of about 50 pores per square micron found for all the mature eggs so far studied as well as the calculated value of about $20 \%$ for the relative pore area are in the range of corresponding values obtained by employing comparable isolation methods for the nuclear envelopes of other kinds of cells such as from rat liver $(24,27)$, rat brain $(25)$, from cultures of human fibroblasts and HeLa cells (46), from dipteran salivary glands (82). These values, however, are markedly lower than those reported for macronuclei of Tetrahymena pyriformis $(86,88)$ and for at least some nuclei of the heterogeneous nuclear populations isolated from pea seedlings (92) and onion roots (23). They are also markedly below those found in the annulate lamellae of the same amphibian oocytes, which contain up to 175 pores per square micron and thus reveal about $35-50 \%$ of their cisternal area as "open" pore area (68). The deviation of the relatively constant pore frequency of 50 pores per square micron found in our material from the value of 25 pores per square micron counted by Merriam (56) for air-dried nuclear envelopes from mature Rana pipiens eggs can hardly be explained.

\section{DISCUSSION}

From the results of the present study it is evident that the appearance of the nuclear envelope of amphibian oocytes as has been shown by Gall $(33,34)$ does not reflect the true pore complex structure, but merely one of the several structural aspects which depend considerably on the special preparation conditions used. Since a series of other contradictory remarks on the ultrastructural details of the nuclear 

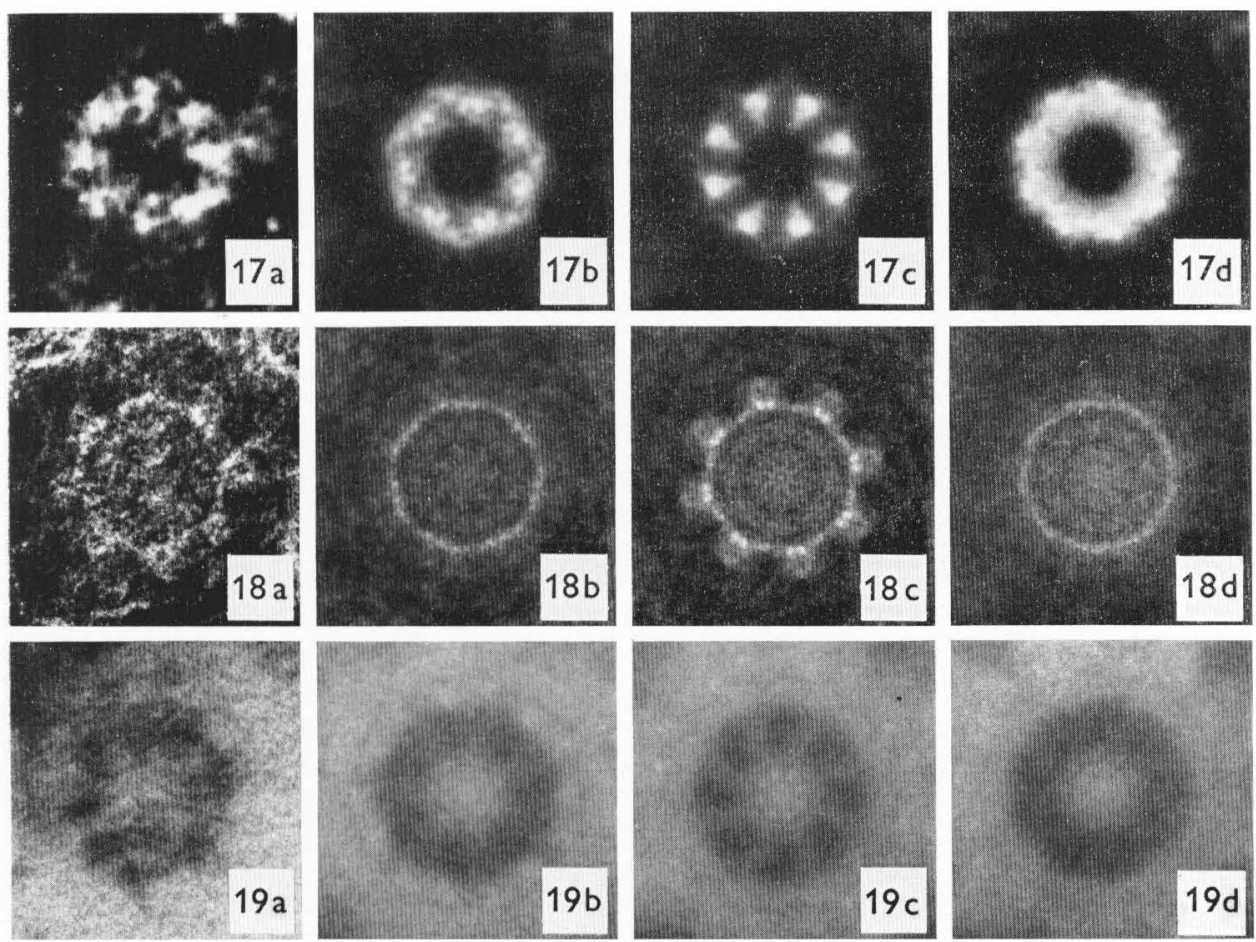

Figs. 17-19. Markham analyses (49) of the annular subunits of the nuclear envelopes from mature Triturus alpestris oocytes reveal a strong eightfold radial symmetry for negatively stained isolated envelopes (Figs. 17 and 18) as well as in tangential sections (Fig. 19). The enhancement of the radial pattern is seen only for $n=8$ (c), but not for $n=7$ (b) and $n=9$ (d) or any other of the values examined, i.e., from $n=5$ to $n=12$. Note that in Fig. 17 there is also an indication of a symmetrical arrangement of the tip-like conical projections extending from the annulus into the pore lumen. All $\times 160,000$.

pore complex exists, particularly what concerns the amphibian oocyte, it might be most valuable to discuss the individual pore complex constituents separately.

1. The membranous part of the pore complex, i.e., the "naked" pore as such, includes not only the anticlinal membrane cylinder of the cisternal discontinuity but also a certain small portion of the adjacent cisternal sheet. This is clearly demonstrated by the fragmentation pattern of the nuclear envelope (Fig. 3), which shows that a small piece of the immediately surrounding membrane belongs to the ultimate porecontaining units, i.e., the isolated pore complexes. This fact, which can be observed also in micrographs of other authors from diverse animal and plant cell nuclei (25, $46,57,92)$ and also from the porous cisternae of the annulate lamellae $(68)$, demonstrates that the pore complexes represent remarkably stable parts of the envelope (see also 65). Such typical fragments have been regarded by Monroe et al. (57) as an 

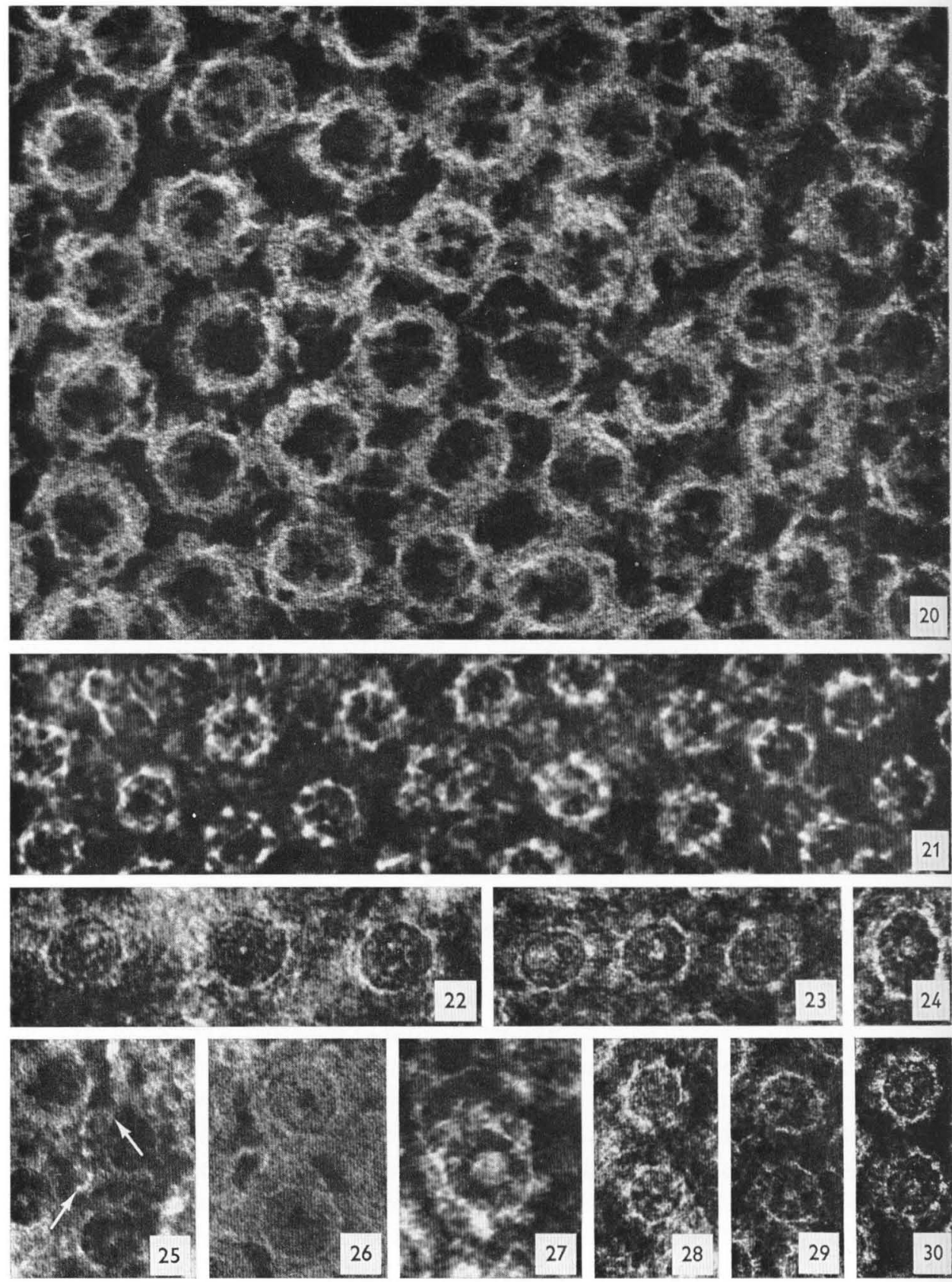

FIGS. 20-30. Ultrastructural details of the pore complexes of nuclear envelopes isolated from mature amphibian oocytes. In Fig. 20 the annulus-attached fibrils can be seen as interconnecting neighboring pore complexes. Similar fibrils can also be discerned within the inner part of the pore in Fig. 21 , where also the local differences in the state of preservation of annular material are obvious. In Figs. 22 and 25 these fibrils seem to be associated with granules (arrows in Fig. 25 denote such a 

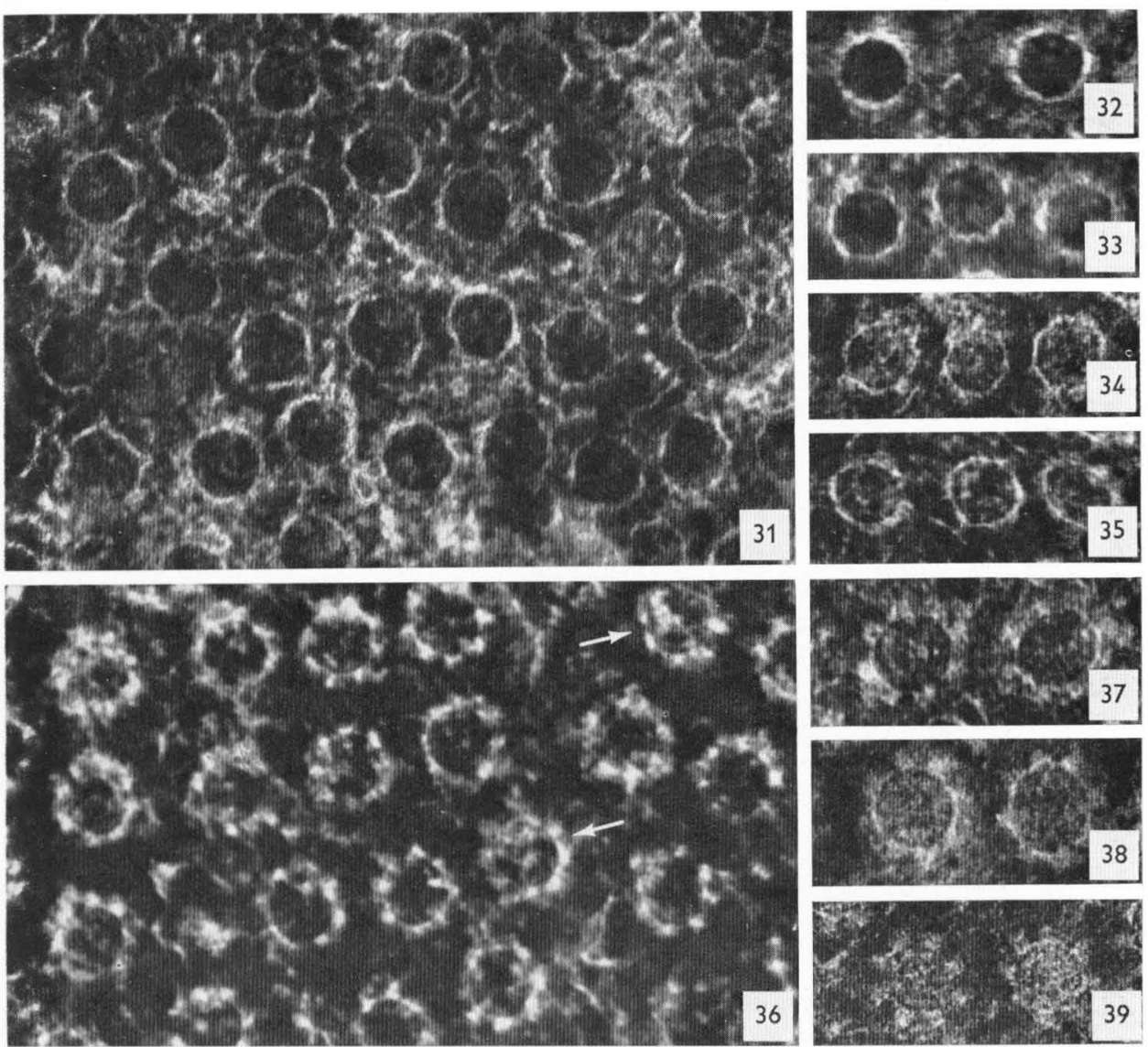

FIGS. 31-35. Diversity of pore shape in negatively stained oocyte nuclear envelopes from different amphibian species. In Fig. 31 circular pore outlines are observed besides more polygonal and even rather oddly distorted ones. Typical pore-shaped deviations from the circularity (Fig. 32) can be seen from Fig. 33 (slightly polygonal), Fig. 34 (polygonal, the pore at the right, e.g., is octagonal), and Fig. 35 (pear-shaped). All $\times 100,000$.

FIGs. 36-39. Differences in the presence and appearance of the annular granules. In Fig. 36 some of the pores reveal annular subunits while others do not. Note also internal pore complex fibrils (arrows). Fig. 37 shows circular pores with well recognizable annular subunits lying at the pore margin. In Fig. 38 such annular granules are seen as lying at the angles of an polygon (right pore). In Fig. 39 the eight annular subunits are the only structures recognizable in the pore complex, and the pore circumference is poorly detectable. Fig. 36, ×110,000; Fig. 37, × 125,000; Figs. 38 and 39, $\times 110,000$.

granule-studded fibril). Variability in the dimensions of the central granules is evident from Figs. 22-24 and Fig. 27. The range of such central granule diameters is from about $40 \AA$ (e.g., the median pore in Fig. 22) up to more than $100 \AA$ (Figs. 23 and 24) and in a few cases even to about $250 \AA$ (Fig. 27). The composed structure of this granule and the "inner ring" fibrils can be detected in Figs. 23 (median pore) and 24. Fig. 26 shows the "central channel" in the center of the pore com plex, i.e., a PTA-filled hole left by the diffuse material within the inner part of the pore. Conical projections from the pore margin into the lumen are visible in the lower pore of Fig. 28. The lower pore in Fig. 29 reveals the spoke-like pattern of radiating fibrils connecting the central granule to the pore margin while Fig. 30 depicts pores in which some of their annular subunits have disappeared. Fig. 20, $\times 160,000$; Fig. 21, $\times 120,000$; Fig. 22, $\times 140,000$; Fig. 23, $\times 130,000$; Fig. 24, $\times 160,000$; Fig. 25, $\times 110,000$; Fig. 26, $\times 140,000$; Fig. 27, $\times 170,000$; Figs. 28-30, all $\times 110,000$. 
indication that the pore complex "itself has sufficient rigidity to stand alone." These authors expressed furthermore the plausible conjecture that the observed structural maintenance of the isolated pore complex might be due to the skeletonizing annular subunit globules.

Gall has recently stated that the nuclear pore shape, i.e., the outline of the porelimiting membrane wall, should be octagonal, not circular $(33,34)$. Some authors have confirmed this $(1,43)$ while others have denied it $(24,68,85)$. In our opinion, deviations from the circular pore shape, such as polygonality, are caused by distortion during dehydration and spreading processes and do not represent the in vivo state, as might also be concluded from images like that shown in Figs. 31-35. The reason that octagonality is the most prevalent type of polygonality seems to be easily explained by the structural stability which during the distortion or shrinkage is effected by the symmetrically distributed eight annular granules. Furthermore, perhaps one of the most convincing arguments for an in vivo circularity of the nuclear pores is the observation that freeze-etching studies have so far revealed circular pores (e.g., 9, 10, $47,58,63)$.

2. The granular subunits of the annulus lie in a symmetrical arrangement upon the pore margin on both the nucleoplasmic and the cytoplasmic face. Some remarks that such a regular distribution of annular subunits is present only on the cytoplasmic side ( $I$ ) are apparently disproved by careful analysis of micrographs showing perpendicularly sectioned pore complexes as presented by various authors who depict the electron dense globules on both rims of the pores of plant (e.g., 18,27, and animal cells (e.g., 35, 62). The micrographs of Gall (34), showing no negatively stained annular granules were most likely taken from nuclear envelopes in which the poreassociated material had been detached, as suggested by other remarks in this article.

It is now well established for eukaryotic cells that both the annuli of the pore complex are composed of eight, in some instances nine $(23,24)$ distinct granular subunits distributed in a radial symmetry on the pore rim $(1,18,22-25,46,51,57$, $61,62,77,81,92,93)$. These subunits are seen in negative staining preparations as prominent, staining-repellent particles. The interpretation of earlier authors of these subunits as tubular microcylinders (84) cannot be maintained considering the more recent work on this topic. However, in spite of the extended knowledge of the structure of these annular particles, their biochemical nature and functional meaning is totally unknown. Their dimensions are slightly below that of cytoplasmic ribosomes, and their affinity to the usual uranyl acetate and lead citrate staining also is somewhat different (e.g., Fig. 40; cf 57, 81). Furthermore, they are different from ribosomes in that they usually take up the stain only in their coat, thus leaving a "light core" in sections (cf. micrographs in 13). Some authors report them to be sensitive to RNase (52; see, however, $7,16,55)$ and against trypsin $(16,55)$ and pepsin $(7)$, but the 

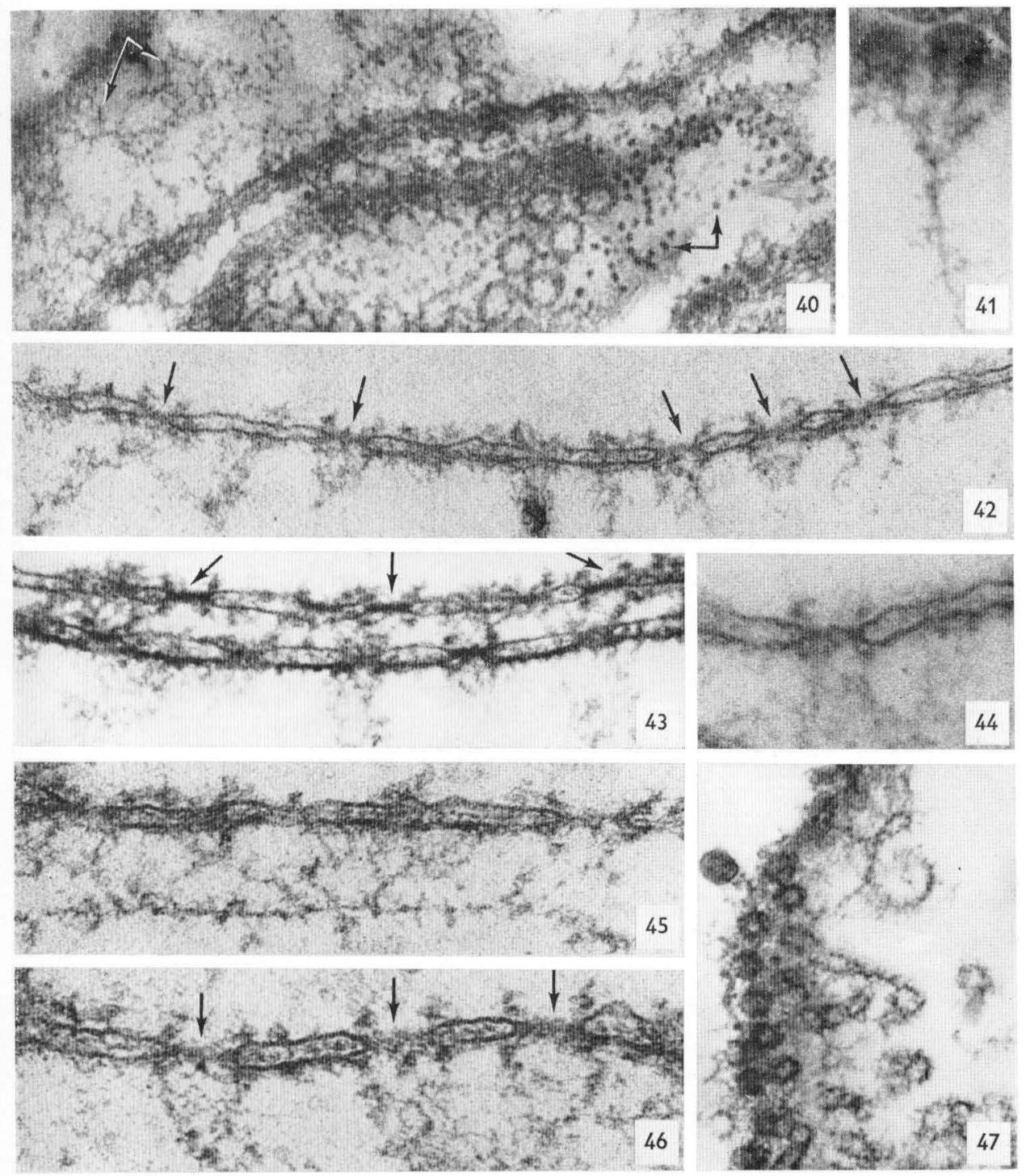

FIGs. 40-47. Sections through or grazing the nuclear envelope of mature oocytes of Triturus alpestris.

Fig. 40 presents an obliquely grazing section to an outpocketing of the nuclear envelope. Note the difference in stainability between the ribosomes upon the outer membrane (arrows) and the annular material and also the finely fibrillar strands in the upper left (arrows). $\times 64,000$.

In Fig. 41 a strand of an annulus-associated fibril branches into subfibrils directed toward the annuli of two neighbor pore complexes. $\times 82,000$.

Fig. 42 shows a survey of the nuclear envelope cisterna. Arrows denote pore complexes which frequently reveal their annular globules lying upon the pore margin. $\times 75,000$.

Fig. 43 documents the similarity of the pore complexes of nuclear envelope to those of the annulate lamellae (arrows). $\times 80,000$.

Fig. 44 shows the relative position of the annular material to the membrane limiting the pore complex at higher magnification. $\times 120,000$.

In Figs. 45 and 46 the intracisternal dense material, which sometimes appears as a lamellar sheet (Fig. 45), can be recognized. Note also the strand-like material which is preferentially associated with the inner annulus of the pore complexes in Fig. 46 (arrows). Both, $\times 100,000$.

Fig. 47 presents a section obliquely grazing the nuclear envelope, thus showing the anastomosing and branching configurations of the annulus-attached fibrils. $\times 42,000$. 
evidence presented, at least for the R Nase digestion, is far from being clear-cut. The annular particles also are reported as sites of special ATPase activity (89-91). As Watson (8I) first noted a close relationship of the annular subunits to the cytoplasmic polysomes in the pore vicinity is obvious in numerous plant and animal cells. Such a spatial relationship has led to the reasonable hypothesis that the annular subunits are parts of forming cytoplasmic polysomes (53). However, there also exist observations which show an electron-transparent, about $250 \AA$ broad area around the annulus as a totally ribosome-free zone (e.g., 18, 61). Furthermore, in some nuclear envelope fractions, for example, from macronuclei of exponentially growing Tetrahymena pyriformis (24) and from onion root tips (23), it has been reported that envelope pieces with well developed annular particles can occur adjacent to others which do not show any material at the pore margins.

3. Thin fibrils attached to the annulus have so far been reported to occur in considerable quantity almost exclusively from oocytes $(39-42,44,73,76)$. They are, nevertheless, observed in some other cells (e.g., 13). An association of these fibrils with granules has been mentioned by Verhey and Moyer (76) for sea urchin eggs and by Kessel and Beams $(40,44)$ for oocytes of diverse origin. Du Praw (16) and Frasca et al. (28) have regarded comparable pore-associated material as chromatin-like. The dimensions of such fibrils, which can be traced in some instances for up to $1 \mu$, their sometimes helical appearance, and the observation that they are connected to the nucleolar periphery in earlier stages of amphibian oogenesis, tempted us to some alternative functional constructions which will be discussed in a following article (26).

4. Fibrils within the pore, however, appear with certainty to be a structural feature common to all kinds of cells (e.g., $27,43,46,77,86-88,92$ ). They can take part in constructing more ordered, compound inner pore structures, such as the "inner ring", i.e., a filamentous circle between pore margin and the pore center (or the central granule, respectively) ot the "spoke-pattern" ("rayons" sensu 77), i.e., a conspicuous radial orientation of the fibrils connecting the central granule and the pore margin.

FIGs. 48-52. Further structural details of the nuclear envelope of mature Triturus alpestris eggs as revealed in sectional work.

Fig. 48 gives a survey of an obliquely tangential section which shows the abundance of the pore complex-attached fibrils in the peripheral nuclear region. Inset at upper left reveals the "spok-like" pattern of radiating internal fibrils connecting the central granule to the annulus. Inset at lower right suggests a helical appearance of the nuclear envelope-attached fibrils. $\times 22,000$; insets, $\times 100,000$.

Fig. 49 presents a crossection through an outpocketing of the nucleus. The pore complex-associated fibrils make up a highly interwoven framework. An about $1 \mu$ long fibrillar strand is observed (arrow) which branches into subfibrils extending toward different pore complexes. $\times 50,000$.

In Fig. 50 such a fibril is shown as terminating at the rim of a nuclear pore. $\times 75,000$.

Fig. 51 shows crossections through the cylindrical "tubes" made up by the fibrils originating from one pore complex. $\times 70,000$.

In Fig. 52 the somewhat helically coiling appearance of branching strands of pore complexassociated fibrils is recognizable. $\times 60,000$. 

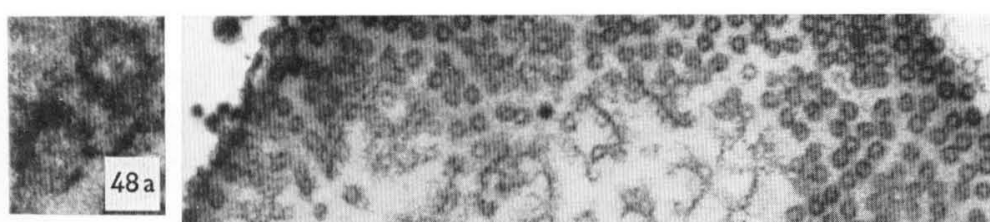

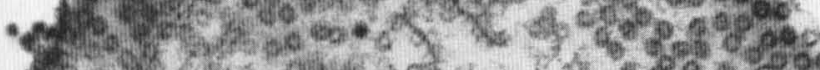

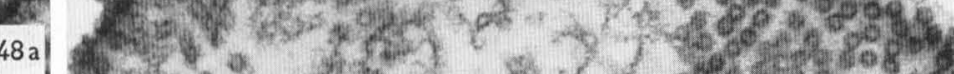

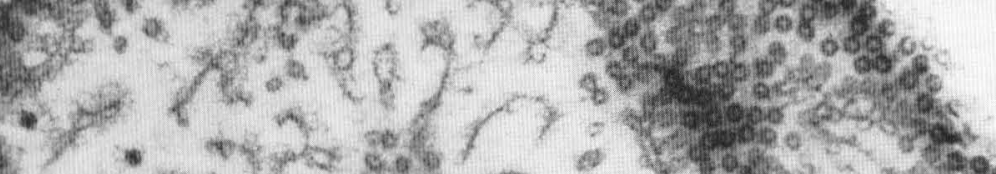

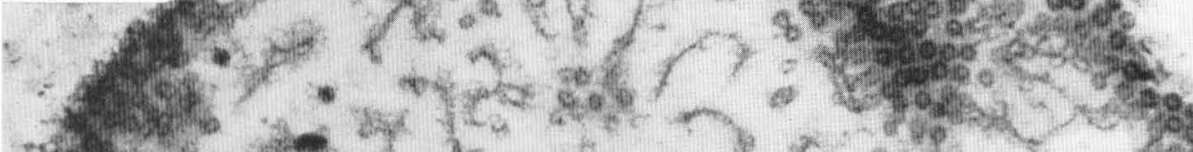

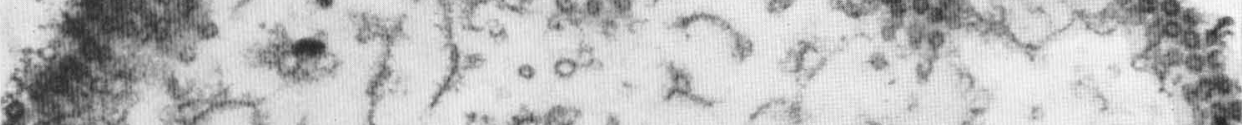

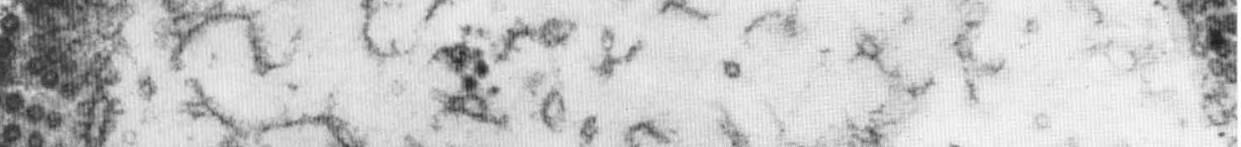

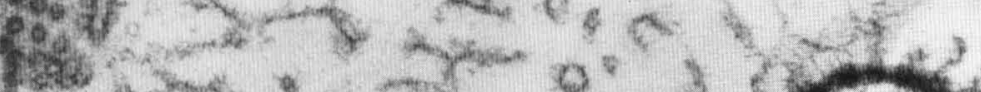
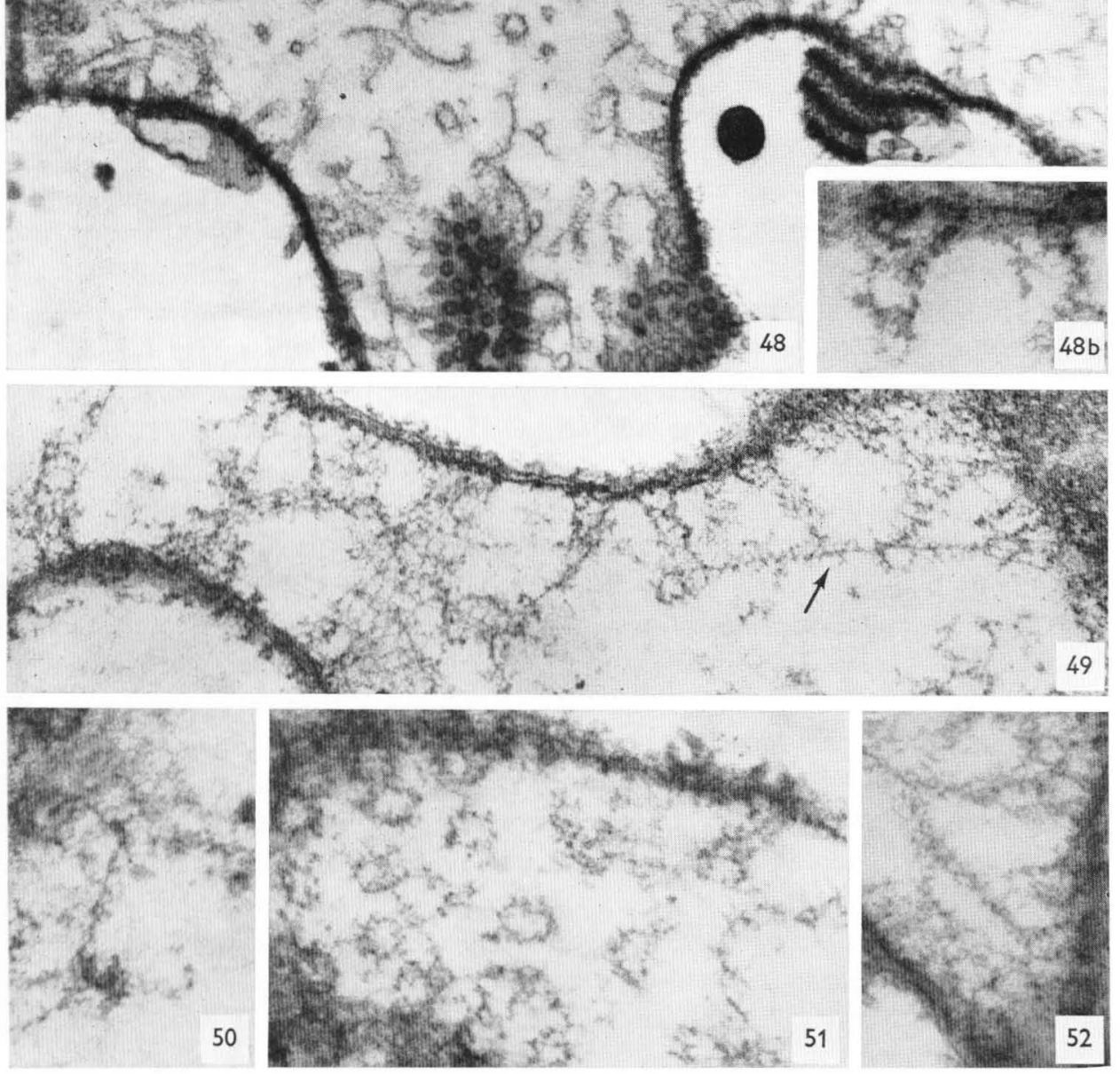
Such a radiating pattern of internal pore fibrils is known to be of widespread occurrence in numerous plant and animal cells from sections as well as from negative staining preparations of isolated envelopes $(13,27,77)$. Similar to the annulusattached fibrils, the internal pore fibrils can frequently be seen also associated with granular particles. Considering the structural similarity of both internal and annulusassociated fíbrils, one gains the impression that these fibrillar structures are identical in chemical composition and biological function.

5. The central granule, this long-known $(2,66)$ centrally located "pore plug", has attracted the attention of many authors. It can be detected in animal and plant nuclei in perpendicular and tangential sections to pore complexes as well as in shadowed or negatively stained isolated envelopes.

From the comparative work on nuclear envelopes carried out in our laboratory we might emphasize the evidence which supports a dynamic rather than a stationary constitutional role of this structure.

a. The central granule is not present in all pores of a nucleus or of a certain nuclear surface area. Neighbouring pores are not rarely distinguished by the presence of the central dot, as could be established from negative staining preparations as well as from serial sections.

b. The central granule dimensions can vary from $40 \AA$ up to about $300 \AA$. Also deviations from the precise central location can occur (cf. 26).

c. The percentage of pore complexes containing a central granule varies remarkably in correlation with the nuclear activity, as indicated by comparison of mature and immature amphibian eggs $(26,56)$ and of different physiological states in synchronized cultures of Tetrahymena pyriformis (87). It fits also nicely into this concept that cells very active in metabolism, for example, dipteran salivary glands $(48,82)$, exponentially growing HeLa cells (46) reveal in general a higher central granule frequency.

d. The central granule frequency is markedly decreased after application of actinomycin D (87).

e. Detailed studies on the substructure of the central particle indicate that it might include a number of different structural phenomena which have only in common that they represent some dense matter in the central part of the pore (compare 26, 43). In some pores the central granule can be recognized as composed of distinct smaller particles $(42,92)$. There exist numerous indications from which it appears justified to discuss central particles within the pore as representing ribonucleoprotein material during the transit from the nucleus into the cytoplasm (e.g., 3, 8, 21, 26, 39, 44, 71, $73,74,87)$.

6. The amorphous material within the annular doughnuts as well as within the inner part of the pore appears to be the material most easily detachable (see also 1), as indicated by the sequence of pore complex dismantling described in Results. It might 


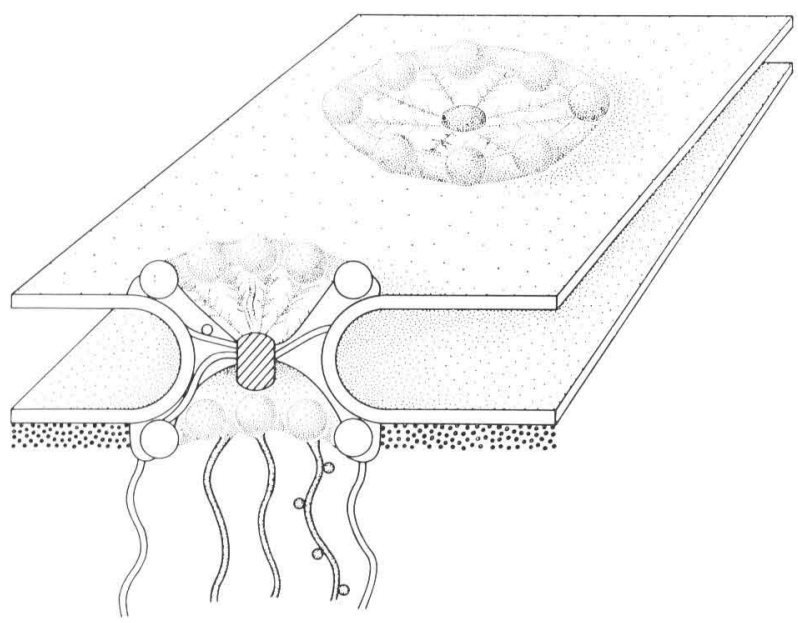

FIG. 53. Diagram illustrating the structural components of the nuclear pore complex of the amphibian oocyte. Annuli lie upon the cytoplasmic and nucleoplasmic margin of the nuclear pore which consists of eight symmetrically distributed granules and some amorphous material. Amorphous material extends also into the pore interior in which the central granule and the internal fibrils appear as particulate structures. Similar fibrils, often studded with small granules, are attached to the nucleoplasmic annulus. Peripheral chromatin underlies the inner nuclear membrane.

be noteworthy to emphasize that this material can withstand even long-term isolation procedures if a proper isolation medium is used $(27,45)$. The material within the pore opening often appears in cross sections, as well as in some shadowed preparations of nuclear fragments (16), so dense that many authors interpreted this matter as a membrane-equivalent diaphragm. Clumps of amorphous material within the pore opening which apparently are firmly attached to the pore margin or the annular subunits, respectively, are more persistent against the isolation procedures and thus give the image of the "tip-like projections."

7. The more or less highly pronounced electron-dense layer beneath the inner nuclear membrane is interrupted at the pore complexes $(19,77)$. Since peripheral accumulations of chromatin, often referred to as heterochromatin, are known from a great many cell types (15) and interaction of the chromosomal nucleohistones with the inner nuclear membrane might be a prerequisite for initiating the DNA replication $(14,50,75)$, we would tentatively consider this thin dense layer of the oocyte nucleus to be some sort of peripheral chromatin. This deoxyribonucleoprotein nature of material underlying the inner nuclear membrane would also explain the selective DNase sensitivity reported from a diatom by Esser (17).

A combination of the structural components summarized in the foregoing leads to an advanced model of the nuclear pore complex (Fig. 53). This diagram summarizes 
our concept of the pore complex and illustrates all described structures with the exception of the "inner ring," whose general existence does not seem yet to be sufficiently established. The possible nature and functional meaning of the pore complex constituents will be discussed in another article, dealing with the nuclear envelopes from earlier stages of oogenesis (26).

The authors thank Miss Sigrid Krien and Miss Marianne Winter for skillful technical assistance. The work was supported by the Deutsche Forschungsgemeinschaft.

\section{REFERENCES}

1. Abelson, H. T. and Smith, G. H., J. Cell Biol. 39, 3A (1968).

2. Afzelius, B. A., Exptl. Cell Res. 8, 147 (1955).

3. Allen, E. R. and Cave, M. D., Z. Zellforsch. Mikroskop. Anat. 92, 477 (1968).

4. Anderson, E. and Beams, H. W., J. Biophys. Biochem. Cytol. 2, Suppl., 439 (1956).

5. André, J. and Roulller, C., Proc. Stockholm Conf. Electron Microscopy 1956, p. 162. Academic Press, New York, 1967.

6. Barnes, B. G. and Davis, J. M., J. Ultrastruct. Res. 3, 131 (1959).

7. Beaulaton, J., Z. Zellforsch. Mikroskop. Anat. 89, 453 (1968).

8. Beermann, W., J. Exptl. Zool. 157, 49 (1964).

9. Branton, D., Proc. Natl. Acad. Sci. U.S. 55, 1048 (1966).

10. Branton, D. and Moor, H., J. Ultrastruct. Res. 11, 401 (1964).

11. Callan, H. G. and Tomlin, S. G., Proc. Roy. Soc. London B137, 367 (1950).

12. Callan, H. G. and Lloyd, L., Phil. Trans. Roy. Soc. London 243, 135 (1960).

13. Claude, A., in Emmelot, P. and Mühlbock, O. (Eds.), Cellular Control Mechanisms and Cancer, p. 41. Elsevier, Amsterdam, 1964.

14. Comings, D. E. and Kakefuda, T., J. Mol. Biol. 33, 225 (1968).

15. Davies, H. G., J. Cell Sci. 3, 129 (1968).

16. DuPraw, E. J., Proc. Natl. Acad. Sci. U.S. 53, 161 (1965).

17. ESSER, K., Experientia 24, 61 (1968).

18. Falk, H. and Kleinig, H., Arch. Mikrobiol. 61, 347 (1968).

19. Fawcett, D. W., Am. J. Anat. 119, 129 (1966).

20. Feldherr, C. M., J. Cell Biol. 20, 188 (1964).

21. — ibid. 25, 43 (1965).

22. Fisher, H. W. and Cooper, T. W., Exptl. Cell Res. 48, 620 (1967).

23. Franke, W. W., J. Cell Biol. 31, 619 (1966).

24. Z Z. Zellforsch. Mikroskop. Anat. 80, 585 (1967).

25. Franke, W. W. and Kartenbeck, J., Experientia 25, 396 (1969).

26. Franke, W. W. and Scheer, U., J. Ultrastruct. Res., 30, 317 (1970).

27. Franke, W. W. and Scheer, U., in preparation.

28. Frasca, J. M., Auerbach, O., Parks, U. R. and Stoeckenius, W., Exptl. Mol. Pathol. 6, 261 (1967).

29. Gall, J. G., Exptl. Cell Res. 7, 197 (1954).

30. - J. Biophys. Biochem. Cytol. 2, Suppl., 393 (1956).

31. ibid. 6, 115 (1959). 
32. Protoplasmatologia 5, 4 (1964).

33. — J. Cell Biol. 27, 121 A (1965).

34. ——ibid. 32, 391 (1967).

35. JACOB, J. and Jurand, A., Chromosoma 14, 451 (1963).

36. Kemp, N. E., J. Biophys. Biochem. Cytol. 2, 281 (1956).

37. Kessel, R. G., J. Cell Biol. 19, 391 (1963).

38. — ibid. 24, 471 (1965).

39. — J. Ultrastruct. Res. 15, 181 (1966).

40. — J. Cell Biol. 36, 658 (1968).

41. — J. Ultrastruct. Res. 22, 63 (1968).

42. — ibid. Suppl. 10 (1968).

43. — Z. Zellforsch. Mikroskop. Anat. 94, 441 (1969).

44. Kessel, R. G. and Beams, H. W., J. Cell Biol. 39, 735 (1968).

45. Kleinig, H., in preparation (1969).

46. Kleinig, H., Comes, P. and Franke, W. W., in preparation (1969).

47. LeAK, L. V., J. Ultrastruct. Res. 25, 253 (1968).

48. McGregor, H. C. and Mackie, J. B., J. Cell Sci. 2, 137 (1967).

49. Markham, R. S., Frey, S. and Hills, G. J., Virology 20, 88 (1963).

50. Marvin, D. A., Nature 219, 485 (1968).

51. Maul, G., J. Cell Biol. 39, 88A (1968).

52. Mentre, P., J. Microscopie 8, 51 (1969).

53. Mepham, R. H. and Lane, G. R., Nature 221, 288 (1969).

54. Merriam, R. W., J. Biophys. Biochem. Cytol. 6, 353 (1958).

55. — ibid. 11, 559 (1961).

56. — J. Cell Biol. 12, 79 (1962).

57. Monroe, J. H., Schidlovsky, G. and Chandra, S., J. Ultrastruct. Res. 21, 134 (1967).

58. Moor, H. and Mühlethaler, K., J. Cell Biol. 17, 609 (1963).

59. MunN, E. A., J. Ultrastruct. Res. 25, 362 (1968).

60. Muscatello, U. and Horne, R. W., J. Ultrastruct. Res. 25, 73 (1968).

61. Muscatello, U., Pasquali-Ronchetti, I. and Barosa, A., J. Ultrastruct. Res. 23, 44 (1968).

62. Nørrevang, A., Vidensk. Medd. Dansk Naturh. Foren. 128, 1 (1965).

63. Northcote, D. H. and Lewis, D. R., J. Cell Sci. 3, 199 (1968).

64. Ornstein, L., J. Biophys. Biochem. Cytol. 2, Suppl., 351 (1956).

65. Pankratz, H. S., J. Cell Biol. 35, 155A (1967).

66. Pollister, A. W., Gettner, U. and Ward, R., Science 120, 789 (1954).

67. Rogers, M. E., J. Cell Biol. 36, 421 (1968).

68. Scheer, U. and Franke, W. W., J. Cell Biol. 42, 519 (1969).

69. Sichel, G., Cellule 66, 97 (1966).

70. Sitte, P., Naturwissenschaften 49, 402 (1962).

71. Stevens, A. R., in Goldstern, L. (Ed.), The Control of Nuclear Activity, p. 189. PrenticeHall, Englewood Cliffs, New Jersey, 1967.

72. Stevens, B. J. and Swift, H., J. Cell Biol. 31, 55 (1966).

73. Swift, H., J. Biophys. Biochem. Cytol. 2, Suppl., 415 (1956).

74. ТАКАмото, T., Nature 211, 772 (1966).

75. Tokuyasu, K., Madden, S. C. and Zeldis, L. I., J. Cell Biol. 39, 630 (1968). 
76. Verhey, C. A. and Moyer, F. H., J. Exptl. Zool. 164, 195 (1967).

77. Vivier, E., J. Microscopie 6, 371 (1967).

78. Ward, R. T. and Ward, E., J. Cell Biol. 39, 139A (1968).

79. Wartenberg, H., Z. Zellforsch. Mikroskop. Anat. 58, 427 (1962).

80. Watson, M. L., J. Biophys. Biochem. Cytol. 1, 257 (1955).

81. — ibid. 6, 147 (1959).

82. Wiener, J., Spiro, D. and Loewenstein, W. R., J. Cell Biol. 27, 107 (1965).

83. Wilkins, M. H. F., Pubbl. Staz. Zool. Napoli 23, Suppl., 109 (1951).

84. Wischnitzer, S., J. Ultrastruct. Res. 1, 201 (1958).

85. Wood, R. L., J. Cell Biol. 31, 125A (1966).

86. Wunderlich, F., Exptl. Cell Res., 56, 369 (1969).

87. — Z. Zellforsch. Mikroskop. Anat.., in press.

88. Wunderlich, F. and Franke, W. W., J. Cell Biol. 38, 458 (1968).

89. Yasuzumi, G. and Tsubo, I., Exptl. Cell Res. 43, 281 (1966).

90. Yasuzumi, G., Nakai, Y., Tsubo, I., Yasuda, M. and Sugioka, T., Exptl. Cell Res. 45, 261 (1967).

91. Yasuzumi, G., Tsubo, I., Okada, K., Terawaki, A. and Enomoto, Y., J. Ultrastruct. Res. 23, 321 (1968).

92. Yoo, B. Y. and BAyLeY, S. T., J. Ultrastruct. Res. 18, 651 (1967).

93. De Zoeten, G. A. and GaArd, G., J. Cell Biol. 40, 814 (1969). 